\title{
STABLE MEASURES AND CENTRAL LIMIT THEOREMS IN SPACES OF STABLE TYPE'
}

\author{
BY
}

\author{
MICHAEL B. MARCUS AND WOJBOR A. WOYCZYNSKI ${ }^{2}$
}

\begin{abstract}
Let $X$ be a symmetric random variable with values in a quasinormed linear space $E$. $X$ satisfies the central limit theorem on $E$ with index $p, 0<p<2$, if $\mathcal{L}\left(n^{-1 / p}\left(X_{1}+\cdots+X_{n}\right)\right)$ converges weakly to some probability measure on $E$. Hoffman-Jorgensen and Pisier have shown that Banach spaces of stable type 2 provide a natural environment for the central limit theorem with index $p=2$. In this paper we show that, for $0<p<2$, quasi-normed linear spaces of stable type $p$ provide a natural environment for the central limit theorem with index $p$. A similar result holds also for the weak law of large numbers with index $p$.
\end{abstract}

0. Introduction. Let $X$ be a symmetric random variable with values in a quasi-normed linear space $E$. We say that $X$ satisfies the central limit theorem on $E$ with index $p, 0<p \leqslant 2$ (in short $\left.X \in \mathrm{CLT}_{p}\right)$, if $\mathcal{L}\left(n^{-1 / p}\left(X_{1}\right.\right.$ $\left.+\cdots+X_{n}\right)$ ) converges weakly to some probability measure, say $\nu$, on $E$. Here $X_{1}, X_{2}, \ldots$ are independent copies of $X$. It is known (see, e.g., [3, Theorem 2]) that such limiting measures $\nu$ must be stable. If we want to specify the limit measure $\nu$ for an $X \in \mathrm{CLT}_{p}$ we say that $X$ is in the domain of normal attraction of $\nu$.

Hoffman-Jørgensen and Pisier [9] have shown that there is a special relationship between CLT $_{2}$ and spaces of stable type 2. That is, the conditions $E X=0, E\|X\|^{2}<\infty$ (that pertain even when $E$ is the real line) imply $X \in \mathrm{CLT}$ if and only if $E$ is of stable type 2 . This led us to pose the following question: Do spaces of stable type $p$ have a special role in relation to CLT $_{p}$ for $0<p<2$, analogous to the role spaces of stable type 2 play for $\mathrm{CLT}_{2}$ ? The answer to this question is yes. In the case of a Banach space $E$ it is contained in the following theorem:

THEOREM 0.1. Let $0<p<2$. The following properties of a Banach space $E$ are equivalent:

(i) $E$ is of stable type $p$.

Received by the editors July 6, 1977 and, in revised form, January 23, 1978.

AMS (MOS) subject classifications (1970). Primary 60B10; Secondary 60B99.

Key words and phrases. Stable measures, domains of attraction, weak law of large numbers, random integral, quasi-normed space, space of stable type $p$.

${ }^{1}$ This research was supported in part by a grant from the National Science Foundation.

${ }^{2}$ The second author was on leave from the Wroclaw University, Poland, while this research was being carried out. 
(ii) For all random vectors $X$ with values in $E \lim _{a \rightarrow \infty} a^{p} P(\|X\|>a)=0$ iff $\mathcal{L}\left(n^{-1 / p}\left(X_{1}+\cdots+X_{n}\right)\right) \rightarrow \delta_{0}$ weakly as $n \rightarrow \infty$, where $X_{1}, X_{2}, \ldots$ are independent copies of $X$ and $\delta_{0}$ is the probability measure concentrated at the origin.

(iii) Let $\eta_{1}, \eta_{2}, \ldots$ be independent, symmetric, real valued random variables, $\theta$ a real valued stable random variable with characteristic function $\exp \left(-|t|^{p}\right)$, $\theta_{1}, \theta_{2}, \ldots$ independent copies of $\theta$ and $c_{p}=\left[\int_{0}^{\infty}\left((\sin x) / x^{p}\right) d x\right]^{-1}$. Then given any sequence $X_{1}, X_{2}, \ldots \in E$ such that $Y=\Sigma \eta_{i} x_{i}$ converges a.s. we have that

$$
\lim _{a \rightarrow \infty} a^{p} P\left[\left\|\sum_{i \in A} \eta_{i} x_{i}\right\|>a\right]=c_{p} \sum_{i \in A}\left\|x_{i}\right\|^{p}<\infty,
$$

for all subsets $A$ of the positive integers if and only if $Z=\Sigma \theta_{i} x_{i}$ converges a.s. and $Y$ satisfies $\mathcal{L}\left(n^{-1 / p}\left(Y_{1}+\cdots+Y_{n}\right)\right) \rightarrow \mathcal{L}(Z)$ weakly as $n \rightarrow \infty$, where $Y_{1}, Y_{2}, \ldots$ are independent copies of $Y$.

This theorem was announced in [20]. It will be proved in a more general setting as Theorem 5.1. In considering spaces classified into spaces of stable type $p$ for $0<p<1$ it is natural and essential to consider general quasinormed spaces such as $l^{q}, 0<q<1$. Otherwise the classification is meaningless since all Banach spaces are of stable type $p$ for $p<1$. Therefore we do not restrict our attention only to Banach spaces but work in the more general context of quasi-normed linear spaces.

$\S 1$ is devoted to precise definitions of the terms we use and to certain preliminary facts. $\$ 2$ deals with the representation of stable random vectors as random integrals and the relationship between these random integrals and their characteristic functionals. These results indicate why spaces of stable type $p$ are a natural environment for stable measures of exponent $p$. Statement (ii) of Theorem 0.1 is evidently a weak law of large numbers. They will be studied in $\$ 3$. $\$ 4$ is technical. It contains extensions to quasi-normed linear spaces of integrability and comparison theorems that were obtained in [8], [10] on Banach spaces. It also treats the integrability of concave functions of random vectors. In $\$ 5$ we prove Theorem 5.1, the extension of Theorem 0.1 to quasi-normed linear spaces. $\$ 6$ contains more results on stable measures and their domains of normal attraction and deals with some concrete examples.

The value of our results is that they are quite general. Results extending the classical theorems on the real line have been found in finite dimensional spaces by Levy and Gnedenko [6], Rvaceva [28] and on Hilbert spaces by Kuelbs and Mandrekar [17] and Klosowska [13]. In \$6 we use Theorem 3.1 [16] of Kuelbs and Mandrekar. This result played a much more central role in the early stages of our work and helped us to clarify our problem. We gratefully acknowledge the help of the referee. 
1. Preliminaries. Let $E$ be a real, separable, quasi-normed complete linear space equipped with a quasi-norm \|\| , i.e., a function from $E$ to the positive real numbers such that $\|x\|=0$ iff $x=0 ;\|\alpha x\|=|\alpha|\|x\|, \alpha \in R, x \in E$; $\|x+y\|<A(\|x\|+\|y\|)$ where $x, y \in E$ and $A$ is a positive real number. Such a space is pseudo-convex and consequently it also admits an $r$-homogeneous norm \|\|$_{r}, 0<r \leqslant 1$, which is equivalent (topologically) to the quasinorm \| || (see [35]). Therefore, without any loss of generality, we shall assume that there exists an $r, 0<r \leqslant 1$, such that \|\|$_{r} \equiv\|\|^{r}$ satisfies the triangle inequality, i.e., $\|x+y\|_{r} \leqslant\|x\|_{r}+\|y\|_{r}, x, y \in E$. $E^{*}$ will denote the topological dual of $E$ and we shall always assume that $E^{*}$ separates the points of E.

Throughout this paper $\theta$ will denote a real valued stable random variable with characteristic function $E \exp i t \theta=\exp -|t|^{p}$ and $\left\{\theta_{i}\right\}$ independent copies of $\theta$.

Definition 1.1. $E$ is said to be of stable type $p, 0<p<2$, if for each $q<p$ there exists a constant $C$ such that for all integers $n$ and any $x_{1}, \ldots, x_{n}$ $\in E$

$$
\left[E\left\|\sum_{i=1}^{n} \theta_{i} x_{i}\right\|^{q}\right)^{1 / q} \leqslant C\left(\sum_{i=1}^{n}\left\|x_{i}\right\|^{p}\right)^{1 / p} .
$$

Actually by [7], [18] $E$ is of stable type $p$ if there exists a single $q \in[0, p)$ such that the above inequality holds. In the case $q=0$ the left-hand side of (1.1) should be replaced by any of the $L^{0}$-gauges. On the other extreme one can replace the $L^{q}$ norms in Definition 1.1 by the Lorentz norm $\Lambda_{p}$. (For a random vector $X$ with values in $E, \Lambda_{p}(X)=\sup _{t>0} t P^{1 / p}(\|X\|>t)$.)

REMARK 1.1. It is a simple consequence of the closed graph theorem in quasi-normed spaces (Theorem 2.7.I [33] and Theorem 6.1 [7]) that $E$ is of stable type $p$ if and only if for all sequences $x_{1}, x_{2}, \ldots$ of elements of $E$ for which $\Sigma\left\|x_{i}\right\|^{p}<\infty$ the series $\Sigma \theta_{i} x_{i}$ converges a.s. (or equivalently converges in $L^{q}, q<p$ ).

If $E$ is of stable type $p$, and if $\xi_{1}, \ldots, \xi_{n}$ are independent with characteristic functions $\exp -\alpha_{i}|t|^{p}, i=1, \ldots, n$, then for any $x_{1}, \ldots, x_{n} \in E$

$$
\left[E\left\|\sum_{i=1}^{n} \xi_{i} x_{i}\right\|^{q}\right]^{1 / q} \leqslant C\left(\sum_{i=1}^{n} \alpha_{i}\left\|x_{i}\right\|^{p}\right)^{1 / p}
$$

where $q<p$ and $C=C(q)$.

If $q \geqslant 2$ then $l_{q}, L_{q}$ are examples of spaces of stable type 2 . If $0<p<q$ and $0<p<2$ then $l_{q}$ and $L_{q}$ are of stable type $p$ but $l_{p}$ and $L_{p}$ are not of stable type $p$. If $p<1$ then all Banach spaces are of stable type $p$.

A theorem of Maurey and Pisier [22] and Krivine [15] states that a Banach 
space $E$ is of stable type $p, p<2$, if and only if $l_{p}$ is not finitely representable in $E$. This gives a particularly nice, purely geometric characterization of spaces of stable type $p$. Recall that $l_{p}$ is finitely representable in $E$ if for each $\varepsilon>0$ and each $n=1,2, \ldots$ one can find $x_{1}, \ldots, x_{n} \in E$ such that for all $\alpha_{1}, \ldots, \alpha_{n} \in \mathbf{R}$,

$$
\left(\sum_{i=1}^{n}\left|\alpha_{i}\right|^{p}\right)^{1 / p} \leqslant\left\|\sum_{i=1}^{n} \alpha_{i} x_{i}\right\| \leqslant(1+\varepsilon)\left(\sum_{i=1}^{n}\left|\alpha_{i}\right|^{p}\right)^{1 / p} .
$$

Spaces of stable type 1 are precisely those which are Beck-convex [1], [26]. A Banach space $E$ is Beck-convex if there exists an $\varepsilon>0$ and an integer $n$ such that for any $x_{1}, \ldots, x_{n} \in S_{E}$, the unit sphere of $E, \| x_{1} \pm x_{2} \pm \cdots \pm$ $x_{n} \| \leqslant(1-\varepsilon) n$ for some choice of \pm .

On any quasi-normed space $E$ for each $p, 0<p<2$ and $0<q<p$, there exists a constant $C_{1}$ such that for all integers $n$ and $x_{1}, \ldots, x_{n} \in E$

$$
C_{1}\left(\sum_{i=1}^{n}\left\|x_{i}\right\|^{p}\right)^{1 / p}<\left[E\left\|\sum_{i=1}^{n} \theta_{i} x_{i}\right\|^{q}\right]^{1 / q}
$$

(see [21]).

We shall also refer to spaces of Rademacher type $p, 0<p<2$. $E$ is said to be of Rademacher type $p, 0<p<2$, if there exists a constant $C_{2}$ such that for all integers $n$ and any $x_{1}, \ldots, x_{n} \in E$

$$
\left[E\left\|\sum_{i=1}^{n} \varepsilon_{i} x_{i}\right\|^{p}\right)^{1 / p} \leqslant C_{2}\left(\sum_{i=1}^{n}\left\|x_{i}\right\|^{p}\right)^{1 / p}
$$

where $\varepsilon_{1}, \ldots, \varepsilon_{n}$ are Rademacher functions, that is independent identically distributed random variables with $P\left(\varepsilon_{1}= \pm 1\right)=\frac{1}{2}$. It follows from (1.4) that if $X_{1}, X_{2}, \ldots$ are symmetric random vectors with values in a space of Rademacher type $p$ then

$$
\left[E\left\|\sum_{i=1}^{n} X_{i}\right\|^{p}\right)^{1 / p} \leqslant C_{2}\left(\sum_{i=1}^{n} E\left\|X_{i}\right\|^{p}\right)^{1 / p} .
$$

For more information on types of normed spaces see [31].

Let the triple $(S, \Sigma, m)$ denote a measurable space with measure $m$ and $L^{p}(S, \Sigma, m ; E)$ the completion of the $(S, \Sigma)$ simple functions with values in $E$ under the quasi-norm

$$
\|f\|_{L^{p}}=\left(\int\|f(s)\|^{p} m(d s)\right)^{1 / p}
$$

We will sometimes abbreviate $L^{p}(S, \Sigma, m ; E)$ by $L^{p}(E)$ or $L^{p}(m ; E)$. 
A random vector $X$ (i.e., a strongly measurable mapping) on a probability space $(\Omega, \mathcal{F}, P)$ with values in $E$, or equivalently its distribution $\mu=\mathcal{L}(X)$, is called symmetric stable or just stable if for any $\alpha, \beta>0$ and independent copies $X_{1}, X_{2}$ of $X$ there exists a $\gamma>0$ such that

$$
\mathcal{L}\left(\alpha X_{1}+\beta X_{2}\right)=\mathcal{L}(\gamma X) \text {. }
$$

In (1.7) we always have $\gamma=\left(\alpha^{p}+\beta^{p}\right)^{1 / p}$ for some $p \in(0,2]$. In this case $X$ and $\mu$ are called stable of exponent $p$.

Since we assume that $E^{*}$ separates points in $E$ an $E$-valued random vector is stable if and only if, for each $x^{*} \in E^{*}, x^{*} X$ is a real stable random variable [5]. In this case the characteristic functional of any stable random vector $X$ has the representation

$$
E \exp i x^{*} X=\exp \left(-\int_{E}\left|x^{*} x\right|^{p} \sigma(d x)\right)
$$

[29], where $0<p \leqslant 2$ is the exponent of $X$ and $\sigma$ is a finite Borel measure on $E$ such that $\int_{E}\|x\|^{p} \sigma(d x)<\infty$. The measure $\sigma$ can always be chosen to be concentrated on the unit sphere thereby satisfying the integrability condition. We shall refer to $\sigma$ as the spectral measure of $X$, or equivalently, $\mu$,

Discrete spectral measures $\Sigma \alpha_{i} \delta_{\left\{x_{i}\right\}}$ correspond to stable random vectors of the form $\Sigma\left|\alpha_{i}\right|^{1 / p} \theta_{i} x_{i}$. In this case, to avoid reduction to lower dimensional cases we assume that

$$
x_{i} \notin \overline{\operatorname{span}}\left[x_{j} ; j=1,2, \ldots ; i \neq j\right],
$$

$i=1,2, \ldots$

A set $K$ is absolutely $r$-convex if, for any $\alpha_{1}, \ldots, \alpha_{n} \in R ; x_{1}, \ldots, x_{n} \in K$, $\Sigma\left|\alpha_{i}\right|^{r}=1$ implies $\sum \alpha_{i} x_{i} \in K$.

2. Random integrals and weak convergence of stable measures on spaces of stable type $p$. We begin with the construction of random integrals of the form $\int f d M$ where $f$ is deterministic and $E$-valued and $M$ is a stable real random measure. For real valued $f$ and even more general $M$ this construction can be found in [2] and [30].

Let $m$ be a nonnegative, finite measure on a measurable space $(S, \Sigma)$ and let $M$ be a stable independently scattered random measure of exponent $p$ on $\Sigma$ for which $m$ is the control measure, that is, for each $A \in \Sigma, E$ exp itM $(A)$ $=\exp \left(-m(A)|t|^{p}\right)$. Recall that a mapping $M: \Sigma \rightarrow L^{0}(\Omega, \mathscr{F}, P)$ is called an independently scattered random measure if for any pairwise disjoint $A_{1}, A_{2}, \ldots \in \Sigma$ the random variables $M\left(A_{1}\right), M\left(A_{2}\right), \ldots$ are stochastically independent and $M\left(\cup A_{i}\right)=\sum M\left(A_{i}\right)$, where the series on the right converges in probability. It follows from Kolmogorov's consistency theorem that given such a control measure $m$ one can construct the related independently scattered random stable measure $M$. 
If $f: S \rightarrow E$ is a simple function, i.e., $f=\Sigma x_{i} I_{A_{i}}$, where $A_{i} \in \Sigma$ are pairwise disjoint and $x_{i} \in A_{i}\left(I_{A}\right.$ denotes the indicator function of the set $\left.A\right)$ we set

$$
\int_{S} f(s) M(d s)=\sum x_{i} M\left(A_{i}\right)
$$

In this case $\int f d M$ is a stable $E$-valued random vector. For $f$ simple and in $L^{p}(S, \Sigma, m ; E)$ and for $q<p$ the map $f \rightarrow \int_{S} f(s) M(d s)$ is a linear operator with values in $L^{q}(\Omega, \mathcal{F}, P ; E)$. By (1.2) we see that

$$
\begin{aligned}
\left\|\int f d M\right\|_{L^{q}} & =\left(E\left\|\sum x_{i} M\left(A_{i}\right)\right\|^{q}\right)^{1 / q} \\
& \leqslant C\left(\sum m\left(A_{i}\right)\left\|x_{i}\right\|^{p}\right)^{1 / p}=C\left(\int\|f(s)\|^{p} m(d s)\right)^{1 / p} .
\end{aligned}
$$

Since the simple functions are dense in $L^{p}(S, \Sigma, m ; E)$ there exists a unique extension of this operator onto the whole of $L^{p}$. This extension will be denoted also by $\int f d M, f \in L^{p}$ and satisfies (2.2). Summarizing we get

THEOREM 2.1. If $E$ is of stable type $p$ and $M$ is a stable independently scattered random measure of exponent $p$ on $(S, \Sigma)$ with control measure $m$, then, for each $q, 0<q<p<2$, there exists a linear map

$$
L^{p}(S, \Sigma, m ; E) \ni f \rightarrow \int_{S} f d M \in L^{q}(\Omega, \mathcal{F}, P ; E)
$$

the values of which are stable random vectors in $E$ satisfying

$$
\left(E\left\|\int f d M\right\|^{q}\right)^{1 / q}<C\left(\int\|f\|^{p} d m\right)^{1 / p}
$$

for some finite constant $C$ independent of $f$.

REMARK 2.1. The inequality (2.4) is also valid if the $L^{q}$ norm is replaced by the Lorentz norm $\Lambda_{p}\left(\int f d M\right)$.

It is quite easy to compute the characteriscic functional of $\int f d M$. Indeed, take simple functions $f_{n}=\Sigma_{i} x_{i}^{n} I_{A_{i}^{n}}$ converging to $f$ in $L^{p}(m ; E)$. Then because of (2.4), $\mathcal{L}\left(\int f_{n} d M\right) \rightarrow \mathcal{L}\left(\int f d M\right)$ weakly. Therefore for each $x^{*} \in E^{*}$

$$
\begin{aligned}
E \exp i x^{*} \int f d M & =\lim _{n} E \exp \left[i x^{*} \sum x_{i}^{n} M\left(A_{i}^{n}\right)\right] \\
& =\lim _{n} \prod_{i} \exp \left(-m\left(A_{i}^{n}\right)\left|x^{*} x_{i}^{n}\right|^{p}\right) \\
& =\lim _{n} \exp \left(-\int\left|x^{*} f_{n}(s)\right|^{p} m(d s)\right) \\
& =\exp \left(-\int_{s}\left|x^{*} f(s)\right|^{p} m(d s)\right) .
\end{aligned}
$$


We use this in the following corollary which, in the particular case $S=E$ and $f(x)=x$, is due to Mouchtari [23].

COROLlaRY 2.2. The following two properties are equivalent:

(2.5) $E$ is of stable type $p, 0<p \leqslant 2$ :

(2.6) For any finite measure space $(S, \Sigma, m)$ and any $f \in L^{p}(S, \Sigma, m ; E)$ the function

$$
E^{*} \ni x^{*} \rightarrow \exp \left(-\int_{S}\left|x^{*} f(s)\right|^{p} m(d s)\right) \in \mathbf{R}
$$

is the characteristic functional of a stable measure $\mu=\mathcal{L}\left(\int f d M\right)$ on $E$.

Proof. Theorem 2.1 and the computation of the characteristic functional of $\mathcal{L}\left(\int f d M\right)$ given above show that (2.5) implies (2.6).

Now, assume (2.6) and let $x_{1}, x_{2}, \ldots$ be a sequence of elements of $E$ such that $\Sigma\left\|x_{i}\right\|^{p}<\infty$. Take $S=E$ with $\Sigma$ being the Borel sets of $E$. Let $m$ be concentrated on $x_{1}, x_{2}, \ldots$ and such that $m\left(\left\{x_{i}\right\}\right)=\left\|x_{i}\right\|^{p}$. Finally take $f(x)=x /\|x\|$. By (2.6), $\varphi\left(x^{*}\right)=\exp \left[-\Sigma\left|x^{*} x_{i}\right|^{p}\right]$ is the characteristic functional of $\mathcal{L}\left(\sum \theta_{i} x_{i}\right)$. Since the summands $\theta_{i} x_{i}$ are symmetric and independent and because for each $x^{*} \in E^{*}$

$$
\lim _{n} \exp \left[-\sum_{i=1}^{n}\left|x^{*} x_{i}\right|^{p}\right]=\varphi\left(x^{*}\right),
$$

by Ito-Nisio's theorem [31, II, p. 274], which extends easily to spaces with $r$-homogeneous norms and separating duals, we obtain the a.s. convergence of the series $\sum \theta_{i} x_{i}$. That means $E$ is of stable type $p$.

REMARK 2.2. In representing all stable measures $\mu$ on spaces $E$ of stable type $p$ we can restrict our attention to measures of the form $\mu=$ $\mathcal{L}\left(\int_{S_{E}} x M(d x)\right)$ where the control measure $m$ of $M$ is concentrated on the unit sphere $S_{E}$ of $E$. This follows by a simple change of variables.

Spaces of stable type $p$ have the property that the weak convergence of spectral measures implies the weak convergence of the corresponding stable measures. The following theorem makes this precise. We will not use this theorem in this paper but we would like to state it here for future reference. A somewhat weaker version was proved for $p=2$ by Rosinski and Woyczynski [32].

THEOREM 2.3. The following conditions are equivalent:

(2.7) $E$ is of stable type $p, 0<p \leqslant 2$.

(2.8) If $\sigma_{1}, \sigma_{2}, \ldots$ are spectral measures such that $\sigma_{i}$ converges weakly to $\sigma_{\infty}$ on $S_{E}$ and if $\mu_{1}, \mu_{2}, \ldots$ are the corresponding stable measures on $E$ then there exists a stable measure $\mu_{\infty}$ and $\mu_{i}$ converges weakly to $\mu_{\infty}$ on $E$. 
Proof. (2.7) implies (2.8). Let $M_{n}, n=1,2, \ldots, \infty$, be stable independently scattered random measures of exponent $p$ on $S_{E}$ with control measures $\sigma_{n}$. Let $\varepsilon>0$. By Prokhorov's theorem we can find a compact set $K \subset S_{E}$ such that, for all $n=1,2, \ldots, \infty ; \sigma_{n}\left(S_{E} / K\right)<\varepsilon$. Let $f=\sum x_{i} I_{A_{i}}$ be simple functions with finite range such that the $A_{i}$ are continuity sets of $\sigma_{\infty}$, $\|f(x)-x\| \leqslant \varepsilon$ on $K$ and $\|f(x)\|<1$ elsewhere. Then

$$
\begin{aligned}
\int_{S_{E}}\|x-f(x)\|^{p} \sigma_{n}(d x)= & \int_{K}\|x-f(x)\|^{p} \sigma_{n}(d X) \\
& +\int_{S_{E} / K}\|x-f(x)\|^{p} \sigma_{n}(d x) \\
< & \varepsilon^{p}+(2 A)^{p} \varepsilon
\end{aligned}
$$

for all $n=1,2, \ldots, \infty$ where $A$ is the constant associated with the quasinorm. By Theorem 2.1, we have that if $q<p$

$$
\begin{aligned}
{\left[E\left\|\int_{S_{E}}(x-f(x)) M_{n}(d x)\right\|^{q}\right]^{1 / q} } & <C\left(\int_{S_{E}}\|x-f(x)\|^{p} \sigma_{n}(d x)\right)^{1 / p} \\
& \leqslant\left(\varepsilon^{p}+(2 A)^{p} \varepsilon\right)^{1 / p}
\end{aligned}
$$

for all $n=1,2, \ldots, \infty$.

Therefore, because $\mu_{n}=\mathfrak{L}\left(\int x M_{n}(d x)\right)$, and

$$
\begin{aligned}
\int x M_{n}(d x)-\int x M_{\infty}(d x)= & \int(x-f(x)) M_{n}(d x)+\int(f(x)-x) M_{\infty}(d x) \\
& +\int f(x) M_{n}(d x)-\int f(x) M_{\infty}(d x),
\end{aligned}
$$

it is sufficient to show that $\mathscr{L}\left(\int_{S_{E}} f(x) M_{n}(d x)\right) \rightarrow \mathscr{L}\left(\int f(x) M_{\infty}(d x)\right)$ weakly as $n \rightarrow \infty$. However, $\int f(x) M_{n}(d x)$ are stable random vectors taking values in a fixed finite dimensional subspace of $E$ spanned by the values of $f$. Therefore to prove weak convergence it is sufficient to prove convergence of the characteristic functionals. For each $x^{*} \in E^{*}$

$$
\begin{aligned}
E \exp \left[i x^{*}\right. & \left.\int f(x) M_{n}(d x)\right]=\exp \left[-\int_{S_{E}}\left|x^{*} f(x)\right|^{p} \sigma_{n}(d x)\right] \\
& =\exp \left[-\sum\left|x^{*} x_{j}\right|^{p} \sigma_{n}\left(A_{j}\right)\right]_{n \rightarrow \infty} \exp \left[-\sum\left|x^{*} x_{j}\right|^{p} \sigma_{\infty}\left(A_{j}\right)\right] \\
& =E \exp \left[i x^{*} \int f(x) M_{\infty}(d x)\right]
\end{aligned}
$$

because $A_{j}$ 's are continuity sets for $\sigma_{\infty}$ and $\sigma_{n} \rightarrow \sigma_{\infty}$ weakly. Clearly the measure $\mu_{\infty}=\mathfrak{L}\left(\int x M_{\infty}(d x)\right)$.

If $E$ is not of stable type $p$ we can find $x_{1}, x_{2}, \ldots, \Sigma_{i-1}^{\infty}\left\|x_{i}\right\|^{p}<\infty$ but $\sum_{i=1}^{\infty} \theta_{i} x_{i}$ does not converge. We take for $\sigma_{\infty}$ the measure concentrated on 
$x_{i} /\left\|x_{i}\right\|$ such that $\sigma_{\infty}\left(\left\{x_{i} /\left\|x_{i}\right\|\right\}\right)=\left\|x_{i}\right\|^{p}, i=1,2, \ldots$, and for $\sigma_{n}$ the measure concentrated on $x_{i} /\left\|x_{i}\right\|, i=1, \ldots, n$ such that $\sigma_{n}\left(\left\{x_{i} /\left\|x_{i}\right\|\right\}\right)=$ $\left\|x_{i}\right\|^{p}, i=1, \ldots, n$. Clearly $\sigma_{n} \rightarrow \sigma_{\infty}$ weakly on $S_{E}$ as $n \rightarrow \infty$. Consider the corresponding stable measures $\mu_{n}, n=1,2, \ldots$ We have $\mu_{n}=\mathcal{L}\left(\sum_{i=1}^{n} \theta_{i} x_{i}\right)$. To say that the $\mu_{n}$ converges weakly is to say that $\sum_{i=1}^{n} \theta_{i} x_{i}$ converges weakly. However, by the extended Ito and Nisio theorem this would imply that $\sum_{i=1}^{\infty} \theta_{i} x_{i}$ converges a.s. and this is not true.

3. Weak law of large numbers. We prove an infinite dimensional analogue of the classical weak law of large numbers of Kolmogorov (see [4]). This result will be important in describing the domain of normal attraction of stable measures. The assumption that $E$ is of stable type $p$, that appears below, is not only sufficient but also necessary as we will see in Theorem 5.1.

THEOREM 3.1. Let $E$ be of Rademacher type $p+\varepsilon, 0<p<2$, for some $\varepsilon>0$ and $X_{1}, X_{2}, \ldots$ independent identically distributed random vectors with values in $E$. Then

$$
\frac{X_{1}+\cdots+X_{n}}{n^{1 / p}} \rightarrow 0
$$

in probability if and only if

$$
n P\left(\left\|X_{1}\right\|>n^{1 / p}\right) \rightarrow 0 .
$$

Note. If $E$ is a Banach space then $E$ is of Rademacher type $p+\varepsilon$, $0<p<2$, for some $\varepsilon>0$ if and only if $E$ is of stable type $p$.

Proof. Define $S_{n}=X_{1}+\cdots+X_{n}$. Condition (3.1) implies (3.2) for $X_{1}$ with values in any quasi-normed linear space $E$. To see this note that because of symmetry $\left\|n^{-1 / p}\left(\sum_{i \neq j} X_{i}+X_{j}\right)\right\|_{r}$ and $\left\|n^{-1 / p}\left(\sum_{i \neq j} X_{i}-X_{j}\right)\right\|_{r}$ are equally distributed. It follows that

$$
P\left(\left\|\frac{S_{n}}{n^{1 / p}}\right\|>2^{1-1 / r} \varepsilon\right)>\frac{1}{2} P\left(\sup _{1<j<n}\left\|\frac{X_{j}}{n^{1 / p}}\right\|>\varepsilon\right) .
$$

Thus (3.1) implies $P\left(\sup _{1<j<n}\left\|X_{j}\right\|>\varepsilon n^{1 / p}\right) \rightarrow 0$ and this is equivalent to (3.2).

We now show that (3.2) implies (3.1). Let $Y_{k}=X_{k} I_{\left[\left\|X_{k}\right\|<n^{1 / p}\right]}, Z_{n}=Y_{1}$ $+\cdots+Y_{n}$ and $B_{n}=\left[S_{n}=Z_{n}\right.$ ]. By assumption (and Theorem 1 [22], [25] in the case of Banach space $E$ ) there exists a $q, p<q<2$, such that $E$ is of Rademacher type $q$. Now, for each $\varepsilon>0$, 


$$
\begin{aligned}
P\left(\left\|S_{n} / n^{1 / p}\right\|\right. & >\varepsilon) \\
& <P\left(B_{n}\right) P\left(\left\|S_{n} / n^{1 / p}\right\|>\varepsilon \mid B_{n}\right)+P\left(B_{n}^{c}\right) P\left(\left\|S_{n} / n^{1 / p}\right\|>\varepsilon \mid B_{n}^{c}\right) \\
& <P\left(\left\|Z_{n} / n^{1 / p}\right\|>\varepsilon\right)+P\left(B_{n}^{c}\right) \\
& <\varepsilon^{-q} E\left\|Z_{n} / n^{1 / p}\right\|^{q}+\sum_{k=1}^{n} P\left(\left\|X_{k}\right\|>n^{1 / p}\right) \\
& <\varepsilon^{-q}-q / p \sum_{k=1}^{n} E\left\|X_{k}\right\|^{q} I_{\left[\left\|X_{k}\right\|<n^{1 / p}\right]}+\sum_{k=1}^{n} P\left(\left\|X_{k}\right\|>n^{1 / p}\right)
\end{aligned}
$$

where at the last step we use (1.5). Continuing, (3.4)

$$
<\varepsilon^{-q} n^{1-q / p} \int_{\left\|X_{1}\right\|<n^{1 / p}}\left\|X_{1}\right\|^{q} d P+n P\left(\left\|X_{1}\right\|>n^{1 / p}\right) .
$$

Thus we need to show that the first term, call it $I_{n}$, goes to zero as $n \rightarrow \infty$. Indeed

$$
\begin{aligned}
I_{n} & =\varepsilon^{-q} n^{1-q / p} \sum_{k=1}^{n} \int_{k-1<\left\|X_{1}\right\|^{p}<k}\left\|X_{1}\right\|^{q} d P \\
& <\varepsilon^{-q} n^{1-q / p} \sum_{k=1}^{n} k^{q / p} P\left(k-1<\left\|X_{1}\right\|^{p}<k\right) \\
& <\varepsilon^{-q} n^{1-q / p} \sum_{k=1}^{n}\left(\sum_{i=1}^{k} i^{q / p-1}\right) P\left(k-1<\left\|X_{1}\right\|^{p}<k\right) \\
& <\varepsilon^{-q_{n} 1-q / p} \sum_{i=1}^{n} i^{q / p-1} P\left(i-1<\left\|X_{1}\right\|^{p}<n\right) \\
& <\varepsilon^{-q_{n} 1-q / p} \sum_{i=1}^{n} i^{q / p-1} P\left(\left\|X_{1}\right\|^{p}>i-1\right) .
\end{aligned}
$$

By (3.2) for each $\delta>0$ we can find an integer $i_{0}$, such that for all $i>i_{0}$, $i P\left(\left\|X_{1}\right\|^{p}>i-1\right)<\delta$. Therefore

$$
\begin{gathered}
I_{n}<\varepsilon^{-q} n^{1-q / p} \sum_{i=1}^{i_{0}} i^{q / p-1} P\left(\left\|X_{1}\right\|^{p}>i-1\right) \\
+\varepsilon^{-q} \delta n^{1-q / p} \sum_{i=i_{0}+1}^{n} i^{q / p-2} .
\end{gathered}
$$

The last term in (3.5) is less than $\left(\delta / \varepsilon^{q}\right) C(p, q)$ where $C$ is a constant that depends only on $p$ and $q$. Since $\delta$ is arbitrary we get $P\left(\left\|S_{n} / n^{1 / p}\right\|>\varepsilon\right) \rightarrow 0$ and this is true for all $\varepsilon>0$. This completes the proof of Theorem 3.1.

As a corollary of the proof of Theorem 3.1 we obtain the following extension of a result which is best possible on the real line (see [24]). 
THEOREM 3.2. Let $E$ be of Rademacher type $p, 0<p<2$, and $X_{1}, X_{2}, \ldots$ independent, symmetric, random vectors with values in $E$. Then

$$
\frac{1}{n} \sum_{i=1}^{n} \int_{\left\|X_{i}\right\|<n}\left\|X_{i}\right\|^{p} d P \rightarrow 0
$$

and

$$
\sum_{i=1}^{n} P\left(\left\|X_{i}\right\|>n^{1 / p}\right) \rightarrow 0
$$

as $n \rightarrow \infty$ implies that

$$
\left(X_{1}+\cdots+X_{n}\right) / n^{1 / p} \rightarrow 0
$$

in probability as $n \rightarrow \infty$. Moreover if $p=2$ then (3.8) implies (3.6) and (3.7).

A Banach space is of stable type 1 if and only if it is Beck-convex. Anticipating Theorem 5.1 we state the following corollary which shows that Beck-convex spaces are exactly those spaces in which an analogue of the classical weak law of large numbers (i.e., $p=1$ ) depends on precisely those conditions necessary on the real line. This is a nice complement to Beck's "strong law of large numbers".

COROLLARY 3.3. Let $E$ be a Banach space; then the following are equivalent: (3.9) $E$ is Beck-convex.

(3.10) For all sequences $X_{1}, X_{2}, \ldots$ of independent, identically distributed, symmetric random vectors with values in $E,\left(X_{1}+\cdots+X_{n}\right) / n \rightarrow 0$ in probability if and only if $n P\left(\left\|X_{1}\right\|>n\right) \rightarrow 0$.

4. Integrability and comparison theorems in quasi-normed spaces. In [8] and [10], Hoffman-Jørgensen and Jain and Marcus obtain results relating the expected value of functions of the norm of the sum of a series of independent Banach space valued random variables to the expected value of the norm of the supremum of the terms of the series. Also in [10] the expected value of convex functions of the norm of the sum of one such series is compared to the expected value of a related series. In $\$ 5$ we will need generalizations of these results in two directions. First we replace the Banach space by a quasi-normed space $E$ and secondly we consider comparison theorems for the expected value of certain concave functions of the quasi-norm.

We do not seek maximum generality but only those results which we use in this paper. Further generalizations can be obtained by our methods.

Let $X_{1}, X_{2}, \ldots$ be independent symmetric random variables with values in $E$. Assume that $S=\sum_{n=1}^{\infty} X_{n}$ exists and define $N=\sup _{n}\left\|X_{n}\right\|_{r}, F(u)=$ $P\left(\|S\|_{r}>u\right), G(u)=P(N>u)$. (Recall that $\|\cdot\|_{r}={ }^{\mathrm{df}}\|\cdot\|^{r}$ is an $r$-homogeneous norm.) 
The next two results are extensions of Lemma 3.4 and Theorem 3.3 in [10]. (Lemma 3.4 [8] is due to Hoffman-Jørgensen.)

LEMMA 4.1. For $\left\{X_{n}\right\}, N, F$ and $G$ as defined above

$$
F(2 t+s) \leqslant G(s)+4 F^{2}(t), \quad t, s>0 .
$$

THEOREM 4.2. Let $\varphi$ be a real valued increasing function satisfying $\varphi(0)=0$ and $\varphi(2 u)<c \varphi(u)$ for some $c>0$. Assume that $\|S\|<\infty$ a.s. Then $E \varphi(N)<$ $\infty$ if and only if $E \varphi\left(\|S\|_{r}\right)<\infty$. Furthermore if $E \varphi\left(\|S\|_{r}\right)<\infty$ then $\lim _{k \rightarrow \infty} E \varphi\left(\left\|\sum_{n=k}^{\infty} X_{n}\right\|_{r}\right)=0$.

The proofs of these lemmas are exactly the proofs given in [10] since the only property of the Banach space that is used in those proofs is that it has a norm and the only property of the norm that is used is that it satisfies the triangle inequality. (Actually all of Theorem 3.3 [10] carries over.)

The extension of a version of the comparison theorems is not so transparent. We begin with a lemma which is analogous to Lemma 5.7 of [10].

LEMMA 4.3. Let $\xi_{1}, \xi_{2}, \ldots$ be independent, symmetric real valued random variables and $x_{1}, x_{2}, \ldots \in E$. Let $\lambda_{1}, \lambda_{2}, \ldots$ be real positive constants such that $\sup _{k}\left|\lambda_{k}\right| \leqslant 1$. Then

$$
E\left(\sup _{n}\left\|\sum_{k=1}^{n} \lambda_{k} \xi_{k} x_{k}\right\|_{r}\right)^{q} \leqslant C_{r, q} E\left(\sup _{n}\left\|\sum_{k=1}^{n} \xi_{k} x_{k}\right\|_{r}\right)^{q}
$$

for $0<q<1$, where $C_{r, q}$ is a constant depending only on $r$, $q$. If $\sum_{k=1}^{\infty} \xi_{k} x_{k}$ converges a.s. in $E$ then also $\sum_{k=1}^{\infty} \lambda_{k} \xi_{k} x_{k}$ converges a.s. and we can replace $\sup _{n}\left\|\sum_{k=1}^{n}\right\|_{r}$ by $\left\|\sum_{k=1}^{\infty}\right\|_{r}$ in (4.1).

Proof. The proof parallels the proof of Lemma 5.7 [10] except that instead of using the convexity of $\varphi$, as in Lemma 5.7 [10], we use the fact that for $0<q \leqslant 1, a_{i} \geqslant 0, i=1, \ldots,\left(\sum a_{i}\right)^{q}<\sum a_{i}^{q}$.

A sequence $\xi_{1}, \xi_{2}, \ldots$ of symmetric real valued random variables is said to be uniformly nondegenerate if there exist constants $a, b>0$ such that

$$
P\left(\left|\xi_{k}\right|>a\right) \geqslant b, \quad k=1,2, \ldots
$$

Also as above let $\varepsilon_{1}, \varepsilon_{2}, \ldots$ denote a Rademacher sequence.

The version of the comparison theorems that we use is:

THEOREM 4.4. Let $\eta_{1}, \eta_{2}, \ldots ; \xi_{1}, \xi_{2}, \ldots$ be two sequences of independent symmetric real valued random variables such that for some $u_{0}>0$

$$
P\left(\left|\eta_{k}\right| \geqslant u\right)<P\left(\left|\xi_{k}\right| \geqslant u\right)
$$

for all $u \geqslant u_{0}$ and all $k=1,2, \ldots$ Also assume that $\xi_{1}, \xi_{2}, \ldots$ is uniformly nondegenerate. Let $x_{1}, x_{2}, \ldots \in E$ and assume that $\sum_{k=1}^{\infty} \xi_{k} x_{k}$ converges a.s. and $E\left\|\sum_{k=1}^{\infty} \xi_{k} x_{k}\right\|^{q}<\infty$ for some $q \leqslant r$. Then $\sum_{k=1}^{\infty} \eta_{k} x_{k}$ converges a.s. and 


$$
E\left\|\sum_{k=1}^{\infty} \eta_{k} x_{k}\right\|^{q} \leqslant C_{1}\left|u_{0}\right|^{q} E\left\|\sum_{k=1}^{\infty} \varepsilon_{k} x_{k}\right\|^{q}+C_{2} E\left\|\sum_{k=1}^{\infty} \xi_{k} x_{k}\right\|^{q}
$$

where $C_{1}, C_{2}$ are constants depending only on $r$ and $q$. Also under these hypotheses $\sum_{k=1}^{\infty} \varepsilon_{k} x_{k}$ converges a.s. and $E\left\|\sum_{k=1}^{\infty} \varepsilon_{k} x_{k}\right\|^{s}<\infty$ for all $s>0$.

Proof. We begin by taking $u_{0}=0$ in (4.3). Following the proof of Lemma $5.10[10]$ we can define $\left\{\eta_{k}\right\}$ and $\left\{\xi_{k}\right\}$ on the same probability space, say $\Omega$, in such a way that $\left|\eta_{k}\right|<\left|\xi_{k}\right|$ a.s.

Let $\left\{\varepsilon_{k}\right\}$ be defined on a probability space $\Omega_{2}$ and consider the space $\Omega_{1} \times \Omega_{2}$. Let $E_{1}$ and $E_{2}$ denote expectations with respect to $\Omega_{1}$ and $\Omega_{2}$. Then for $q^{\prime}=q / r$ and any positive integer $n$

$$
\begin{aligned}
E\left[\left\|\sum_{k=1}^{n} \eta_{k} x_{k}\right\|_{r}^{q^{\prime}}\right] & =E_{1} E_{2}\left\|\sum_{k=1}^{n} \varepsilon_{k}\left|\eta_{k}\right| x_{k}\right\|_{r}^{q^{\prime}}=E_{1} E_{2}\left\|\sum_{k=1}^{n} \varepsilon_{k} \frac{\left|\eta_{k}\right|}{\left|\xi_{k}\right|}\left|\xi_{k}\right| x_{k}\right\|_{r}^{q^{\prime}} \\
& \leqslant C_{r, q^{\prime}} E_{1} E_{2}\left\|\sum_{k=1}^{n} \varepsilon_{k}\left|\xi_{k}\right| x_{k}\right\|_{r}^{q^{\prime}}=C_{r, q^{\prime}} E\left\|\sum_{k=1}^{n} \xi_{k} x_{k}\right\|_{r}^{q^{\prime}}
\end{aligned}
$$

where we use Lemma 4.3 to obtain the inequality. By assumption $\Sigma \xi_{k} x_{k}$ converges a.s. and the sum is in $L^{q}(E)$. Therefore by Theorem 4.2 the series converges also in $L^{q}(E)$ and by completeness of $E$ the above inequality gives the $L^{q}(E)$ convergence of $\Sigma \eta_{k} x_{k}$ and thus (4.4). The almost sure convergence of $\Sigma \eta_{k} x_{k}$ is now an immediate corollary to the well-known fact that in any metrizable group the convergence in probability of a series with independent summands implies its a.s. convergence.

Now, we shall prove the convergence of $\sum \varepsilon_{k} x_{k}$. Let $a$ and $b$ be the constants given in (4.2). Define $\xi_{n}^{\prime}=\xi_{n} I\left(\left|\xi_{n}\right|<a\right)$ and $\xi_{n}^{\prime \prime}=\xi_{n}-\xi_{n}^{\prime}, n=$ $1,2, \ldots$ Because $P\left(\left|\xi_{n}^{\prime}\right| \geqslant u\right) \leqslant P\left(\left|\xi_{n}\right| \geqslant u\right)$ for any $u \geqslant 0$, by the case of the theorem already proved both $\Sigma \xi_{n}^{\prime} x_{n}$ and $\Sigma \xi_{n}^{\prime \prime} x_{n}$ converges a.s. Define independent $\delta_{n}= \pm a$ with probability $b / 2$ and $\delta_{n}=0$ with probability $1-b$. Since $\xi_{n}$ are uniformly nondegenerate we have $P\left(\left|\delta_{n}\right|>u\right)<P\left(\left|\xi_{n}^{\prime \prime}\right|>\right.$ $u$ ) for any $u \geqslant 0$ so that applying the above reasoning once more we obtain the a.s. convergence of $\Sigma \delta_{n} x_{n}$. It now follows from the proof of Theorem 5.7 [7] that $\sum_{k=1}^{\infty} \varepsilon_{k} x_{k}$ converges a.s. (Note that this stage of the proof has nothing to do with a norm and works in a quasi-normed space. In [10], the proof of this stage for $E$ a Banach space makes use of the convexity of the norm, a property not shared by \|\| .)

Once we know that $\sum_{k=1}^{\infty} \varepsilon_{k} x_{k}$ converges a.s. we have

$$
E\left\|\sum_{k=1}^{\infty} \varepsilon_{k} x_{k}\right\|_{r}^{s}<\infty
$$


for all $s>0$. This follows from Theorem 4.2 since $E\left[\sup _{k}\left\|\varepsilon_{k} x_{k}\right\|_{r}^{s}\right]<\infty$.

Define $\rho_{k}=u_{0}\left[-u_{0}\right]$ if $0<\eta_{k}<u_{0}\left[-u_{0} \leqslant \eta_{k}<0\right]$ and 0 otherwise and $\rho_{k}^{\prime}=\eta_{k} I\left(\left|\eta_{k}\right|>u_{0}\right)$. Then $P\left(\left|\eta_{k}\right|>u\right)<P\left(\left|\rho_{k}+\rho_{k}^{\prime}\right|>u\right)$ for all $u>0$ and it follows from the case $u_{0}=0$ that

$$
E\left\|\sum_{k=1}^{\infty} \eta_{k} x_{k}\right\|_{r}^{q^{\prime}} \leqslant C_{r, q^{\prime}}\left[E\left\|\sum_{k=1}^{\infty} \rho_{k} x_{k}\right\|_{r}^{q^{\prime}}+E\left\|\sum_{k=1}^{\infty} \rho_{k}^{\prime} x_{k}\right\|_{r}^{q^{\prime}}\right] .
$$

We have $E\left\|\sum_{k=1}^{\infty} \rho_{k} x_{k}\right\|_{r}^{q^{\prime}} \leqslant\left|u_{0}\right|^{q^{\prime} r} E\left\|\sum_{k=1}^{\infty} \varepsilon_{k} x_{k}\right\|_{r}^{q^{\prime}}$. This inequality follows from the fact that $\left|\rho_{k} / u_{0}\right|<1$, Lemma 4.3 and (4.5). Also since $P\left(\left|\rho_{k}^{\prime}\right|>u\right)<$ $P\left(\left|\xi_{k}\right|>u\right)$ we again use the case $u_{0}=0$ to obtain

$$
E\left\|\sum_{k=1}^{\infty} \rho_{k}^{\prime} x_{k}\right\|_{r}^{q^{\prime}} \leqslant C_{r, q^{\prime}} E\left\|\sum_{k=1}^{\infty} \xi_{k} x_{k}\right\|_{r}^{q^{\prime}}
$$

This completes the proof of the theorem, since the a.s. convergence of the series $\sum \eta_{k} x_{k}$ obtains in exactly the same way as in the case $u_{0}=0$.

5. Domains of attraction of stable laws with discrete spectral measures. This section is devoted to the proof of Theorem 5.1 which shows the essential relationship between the central limit theorem and spaces of stable type $p$. However, some of the preliminary results such as Lemma 5.3 and Theorem 5.4 apply to all quasi-normed spaces and are of independent interest.

TheOREM 5.1. Let $E$ be a quasi-normed linear space, $0<p<2$. For the following properties of $E$ :

(i) $E$ is of Rademacher type $p+\varepsilon$, for some $\varepsilon>0$;

(ii) for all random vectors $X$ with values in $E$,

$$
\lim _{a \rightarrow \infty} a^{p} P(\|X\|>a)=0 \quad \text { iff } \quad \mathcal{L}\left(\frac{X_{1}+\cdots+X_{n}}{n^{1 / p}}\right) \rightarrow \delta_{0}
$$

weakly as $n \rightarrow \infty$, where $X_{1}, X_{2}, \ldots$ are independent copies of $X$ and $\delta_{0}$ is the probability measure concentrated at the origin;

(iii) Let $\eta_{1}, \eta_{2}, \ldots$ be independent, symmetric, real valued random variables, $\theta$ a real valued stable random variable with characteristic function $\exp \left(-|t|^{p}\right)$, $\theta_{1}, \theta_{2}, \ldots$, independent copies of $\theta$ and $c_{p}=\left[\int_{0}^{\infty}\left((\sin x) / x^{p}\right) d x\right]^{-1}$.

Then given any sequence $x_{1}, x_{2}, \ldots$ of elements of $E$ such that $Y=\sum_{i=1}^{\infty} \eta_{i} x_{i}$ exists,

$$
\lim _{a \rightarrow \infty} a^{p} P\left[\left\|\sum_{i \in A} \eta_{i} x_{i}\right\|>a\right]=c_{p} \sum_{i \in A}\left\|x_{i}\right\|^{p}<\infty
$$

for all subsets $A$ of the positive integers, if and only if $Z=\sum_{i=1}^{\infty} \theta_{i} x_{i}$ exists, and $Y$ satisfies 


$$
\mathcal{L}\left(\left(Y_{1}+\cdots+Y_{n}\right) / n^{1 / p}\right) \rightarrow \mathcal{L}(Z)
$$

weakly as $n \rightarrow \infty$, where $Y_{1}, Y_{2}, \ldots$ are independent copies of $Y$;

(iv) $E$ is stable type $p$;

we have the following implications: (i) $\Rightarrow$ (ii) $\Rightarrow$ (iii) $\Rightarrow$ (iv). Furthermore if $E$ is a Banach space, or if $E=l_{r}, 0<r<1$, then (iv) $\Rightarrow(\mathrm{i})$, i.e., all of the above properties are equivalent.

Before proving Theorem 5.1 we obtain a number of preliminary results. The first is a relationship between the tail behaviour of the probability distribution of a symmetric random variable and the behaviour of its characteristic function near zero. It is a special case of an estimate in $[4, p$. 41].

LEMMA 5.2. Let $\eta$ be a symmetric real valued random variable with distribution function $F$ and characteristic function $\Phi$. Then

$$
P\left[|\eta|>\frac{1}{t}\right]<\frac{7}{t} \int_{0}^{t}[1-\Phi(s)] d s .
$$

Let $\eta_{1}, \eta_{2}, \ldots$, and $\theta_{1}, \theta_{2}, \ldots$ be defined as in Theorem 5.1(iii). Recall that a real valued symmetric random variable $\eta$ is said to be in the domain of normal attraction of $\theta$ if $\mathcal{L}\left(n^{-1 / p}\left(\eta_{1}+\cdots+\eta_{n}\right)\right) \rightarrow \mathcal{L}(\theta), n \rightarrow \infty$. A necessary and sufficient condition for $\eta$ to be in the domain of normal attraction of $\boldsymbol{\theta}$ is

$$
\lim _{t \rightarrow 0} \frac{1-\Phi(t)}{|t|^{p}}=1 .
$$

Therefore given $\varepsilon>0$ there exists a $\delta>0$ such that

$$
e^{-(1+\varepsilon)|t|^{p}}<\Phi(t)<e^{-(1-e)|t|^{p}}
$$

for $|t|<\delta$. Also by Theorem 1 [27] we have

$$
\lim _{t \rightarrow \infty} t^{p} P[|\eta|>t]=c_{p}
$$

Let $x_{1}, x_{2}, \ldots$ be elements of $E$. Assume that $Y=\sum_{i=1}^{\infty} \eta_{i} x_{i}$ exists. Let $c_{1}, c_{2}, \ldots$ be an increasing sequence of positive real numbers and define $\eta_{i}=\eta_{i}^{\prime}+\eta_{i}^{\prime \prime}$ where

$$
\eta_{i}^{\prime \prime}= \begin{cases}0, & \left|\eta_{i}\right|<c_{i}, \\ \eta_{i}, & \text { otherwise. }\end{cases}
$$

Similarly we write $Y=Y^{\prime}+Y^{\prime \prime}$ where $Y^{\prime \prime}=\sum_{i=1}^{\infty} \eta_{i}^{\prime \prime} x_{i}$.

Note that $Y^{\prime}$ and $Y^{\prime \prime}$ both converge a.s. This follows by a standard comparison theorem argument. Let $\left\{\eta_{i}\right\}$ be defined on the probability space $(\Omega, \mathscr{F}, P)$ and let $\left\{\varepsilon_{i}\right\}$ be a Rademacher sequence defined on the probability space $\left(\Omega_{1}, \mathscr{F}_{1}, P_{1}\right)$. Consider $\Sigma \varepsilon_{i} \eta_{i} x_{i}$ on $\left(\Omega \times \Omega_{1}, \mathscr{F} \times \mathscr{F}_{1}, P \times P_{1}\right)$. It is 
stochastically equivalent to $\Sigma \eta_{i} x_{i}$ and therefore converges a.s. $\left(P \times P_{1}\right)$. By Fubini's theorem for almost all $\omega \in \Omega(P), \Sigma \varepsilon_{i} \eta_{i}(\omega) x_{i}$ converges a.s. $\left(P_{1}\right)$. Since $\left|\eta_{i}^{\prime}(\omega) / \eta_{i}(\omega)\right|<1$ we have by Lemma 4.3 that $\Sigma \varepsilon_{i} \eta_{i}^{\prime}(\omega) x_{i}$ converges a.s. $P_{1}$ for almost all $\omega \in \Omega(P)$. Another application of Fubini's theorem shows that $\Sigma \varepsilon_{i} \eta_{i}^{\prime} x_{i}$ converges a.s. $\left(P \times P_{1}\right)$ and therefore $\Sigma \eta_{i}^{\prime} x_{i}$ converges a.s. $(P)$. A similar argument shows that $Y^{\prime \prime}$ converges a.s. $(P)$.

$Y^{\prime}$ will be called the truncated part of $Y$, even though $Y^{\prime}$ is not necessarily bounded. Clearly $Y^{\prime}$ depends on $c_{1}, c_{2}, \ldots$ When we speak of all truncated parts $Y^{\prime}$ of $Y$, we mean all the $Y^{\prime}$ terms associated with different admissible sequences $c_{1}, c_{2}, \ldots$ Note that in general $c_{1}, c_{2}, \ldots$ will not be a bounded sequence.

Suppose that $Z=\sum_{i=1}^{\infty} \theta_{i} x_{i}$ exists. Then $Z$ is a stable random vector with values in $E$. We raise the following question. Suppose that each $\eta_{i}$ is individually in the domain of attraction of $\theta$; then under what conditions is $Y$ in the domain of normal attraction of $Z$ ? That is, does

$$
\mathcal{E}\left(\left(Y_{1}+\cdots+Y_{n}\right) / n^{1 / p}\right) \rightarrow \mathcal{E}(Z), \quad n \rightarrow \infty,
$$

where $Y_{1}, Y_{2}, \ldots$, are independent copies of $Y$ ? The next lemma provides an answer to this question. It appears rather abstract but it will prove to be very useful.

LEMMA 5.3. Let $E, \theta,\left\{\theta_{i}\right\},\left\{\eta_{i}\right\},\left\{x_{i}\right\}, Y$, and $Z$ be as above, where each $\eta_{i}$ is in the domain of normal attraction of $\boldsymbol{\theta}$. A necessary and sufficient condition for $Y$ to be in the domain of normal attraction of $Z$ is that all truncations $Y^{\prime}$ of $Y$ are in the domain of normal attraction of $\delta_{0}$.

Proof. We first prove sufficiency. By (5.7), given $\varepsilon>0$, for each $\eta_{i}$ there exists a $c_{i}>0$ such that

$$
c_{p}(1-\varepsilon) / t^{p} \leqslant P\left[\left|\eta_{i}\right|>t\right]<c_{p}(1+\varepsilon) / t^{p}
$$

for $t \geqslant c_{i}$. Based on the sequence $c_{1}, c_{2}, \ldots$ we form the decomposition $Y=Y^{\prime}+Y^{\prime \prime}$, where $Y^{\prime \prime}=\sum_{i=1}^{\infty} \eta_{i}^{\prime \prime} x_{i}$ for $\left\{\eta_{i}^{\prime \prime}\right\}$ as defined in (5.8). Let $\Psi_{i}$ be the characteristic function of $\eta_{i}^{\prime \prime}$. Then

$$
1-\Psi_{i}(s)=2 \int_{c_{i}}^{\infty}\left(\sin \frac{\lambda s}{2}\right)^{2} d F_{i}(\lambda)
$$

where $F_{i}(\lambda)$ is the distribution function of $\eta_{i}$. Therefore we have

$$
1-\Psi_{i}(s) \leqslant c_{p}(1+\varepsilon) / c_{i}^{p} .
$$

Furthermore if $1 / s>c_{i}, \quad 1-\Psi_{i}(s)<-\frac{1}{2} \int_{c_{i}}^{1 / s} \lambda^{2} s^{2} d H_{i}(\lambda)+2 \int_{1 / s}^{\infty} d F_{i}(\lambda)$ where $H_{i}=1-F_{i}$, and 


$$
\begin{aligned}
-\frac{1}{2} \int_{c_{i}}^{1 / s} \lambda^{2} s^{2} d H_{i}(\lambda) & <\left(\frac{c_{p}}{2}\right)\left(\frac{1+\varepsilon}{2}\right)\left(c_{i} s\right)^{2-p_{s} p}+\int_{c_{i}}^{1 / s} s^{2} \lambda H(\lambda) d \lambda \\
& <\left(\frac{c_{p}}{2}\right)(1+\varepsilon)\left[\frac{1}{2}+\frac{1}{2-p}\right] s^{p}<C_{p}^{\prime} s^{p}
\end{aligned}
$$

for some constant $C_{p}^{\prime}$. Also $2 \int_{1 / s}^{\infty} d F_{i}(\lambda)<c_{p}(1+\varepsilon) s^{p}$. Therefore, for $1 / s>$ $c_{i}, 1-\Psi_{i}(s)<C_{p} s^{p}$, where $C_{p}$ is a constant independent of $i$. Combining this with (5.11) we get $1-\Psi_{i}(s)<C_{p} s^{p}$ for all $s>0$. Let $\delta_{p}$ be such that $\left(1-C_{p} s^{p} / n\right)^{n}>1-2 C_{p} s^{p}$ for $|s|<\delta_{p}$. (Clearly $\delta_{p}>0$.) Thus for $|s|<\delta_{p}$

$$
\Psi_{i}^{n}\left(s / n^{1 / p}\right)>\left(1-C_{p} s^{p} / n\right)^{n}>1-2 C_{p} s^{p}
$$

and

$$
1-\Psi_{i}^{n}\left(s / n^{1 / p}\right)<2 C_{p} s^{p} .
$$

By (5.12) and Lemma 5.2, $u_{0}=1 / \delta_{p}$ and $u>u_{0}$. We get

$$
P\left[\left|\frac{\eta_{i, 1}^{\prime \prime}+\cdots+\eta_{i, n}^{\prime \prime}}{n^{1 / p}}\right|>u\right]<C u \int_{0}^{1 / u} 2 C_{p} s^{p} d s<\frac{K_{p} c_{p}}{u^{p}}
$$

for some constant $K_{p}$ independent of $i$. This is the estimate that we will use in the next step of the proof.

By hypothesis $\sum_{i=1}^{\infty} \theta_{i} x_{i}$ exists. Since this implies that $\lim _{i \rightarrow \infty}\left\|\theta_{i} x_{i}\right\|=0$ a.s., it follows from the Borel-Cantelli lemma that $\sum_{i=1}^{\infty}\left\|x_{i}\right\|^{p}<\infty$ (cf. also (1.3)). Following $\$ 4$ let $N=\sup _{i}\left\|\theta_{i} x_{i}\right\|_{r}$. Then

$$
\begin{aligned}
P\left(N>a^{r}\right) & <\sum_{i=1}^{\infty} P\left[\left|\theta_{i}\right|\left\|x_{i}\right\|>a\right] \\
& <\frac{2(1+\varepsilon) \sum_{i=1}^{\infty}\left\|x_{i}\right\|^{p}}{a^{p}} .
\end{aligned}
$$

Thus $E\left(N^{q / r}\right)<\infty$ for $q<p$ and by Theorem 4.2 we also have

$$
E\left\|\sum_{i=1}^{\infty} \theta_{i} x_{i}\right\|^{q}<\infty, \quad q<p
$$

(as we might expect from [8]).

We use Theorem 4.4 together with (5.13) to obtain the essential inequality of this proof, that for any $N, n$ and $q<p \wedge r$

$$
\begin{aligned}
E\left\|\sum_{k=N}^{\infty}\left(\frac{\eta_{k, 1}^{\prime \prime}+\cdots+\eta_{k, n}^{\prime \prime}}{n^{1 / p}}\right) x_{k}\right\|^{q} \\
\quad \leqslant C_{1}^{\prime}\left|u_{0}\right|^{q} E\left\|\sum_{k=N}^{\infty} \varepsilon_{k} x_{k}\right\|^{q}+C_{2}^{\prime} E\left\|\sum_{k=N}^{\infty} \theta_{k} x_{k}\right\|^{q} .
\end{aligned}
$$


Note that in using Theorem 4.4 we compare the random variables ( $\eta_{k, 1}^{\prime \prime}$ $\left.+\cdots+\eta_{k, n}^{\prime \prime}\right) / n^{1 / p}$ with $2 K_{p} \theta_{p}$ in order to satisfy (4.3). The factor $2 K_{p}$ is then absorbed into the constant $C_{2}^{\prime}$. The constants $C_{1}^{\prime}$ and $C_{2}^{\prime}$ depend upon $p$, $q, r$ and $K_{p}$; however all these terms remain fixed throughout what follows.) The existence of the first term on the right is asserted in Theorem 4.4, of the second term on the right in (5.15). It also follows from Theorem 4.2 that both of these terms can be made arbitrarily small by taking $N$ sufficiently large.

We now show that $Y^{\prime \prime}$ is in the domain of normal attraction of $Z$. Since bounded Lipschitz functions of order $\alpha<1$ form an algebra of functions on $E$ which separate points of $E$ by Lemma 3.1 [9] it is sufficient to show that

$$
\lim _{n \rightarrow \infty} E f\left(\sum_{i=1}^{\infty} \frac{\eta_{i, 1}^{\prime \prime}+\cdots+\eta_{i, n}^{\prime \prime}}{n^{1 / p}} x_{i}\right)=E f\left(\sum_{i=1}^{\infty} \theta_{i} x_{i}\right)
$$

for all bounded functions $f$ satisfying $|f(x)-f(y)|<K\|x-y\|^{\alpha}$ for all $x, y \in E$ and some $\alpha \leqslant 1$ ( $K$ is a constant depending on $f$ ). We have

$$
\begin{aligned}
\left|E f\left(\sum_{i=1}^{\infty} \frac{\eta_{i, 1}^{\prime \prime}+\cdots+\eta_{i, n}^{\prime \prime}}{n^{1 / p}} x_{i}\right)-E f\left(\sum_{i=1}^{\infty} \theta_{i} x_{i}\right)\right| \\
\leqslant\left|E f\left(\sum_{i=1}^{\infty} \frac{\eta_{i, 1}^{\prime \prime}+\cdots+\eta_{i, n}^{\prime \prime}}{n^{1 / p}} x_{i}\right)-E f\left(\sum_{i=1}^{N-1} \frac{\eta_{i, 1}^{\prime \prime}+\cdots+\eta_{i, n}^{\prime \prime}}{n^{1 / p}} x_{i}\right)\right| \\
+\left|E f\left(\sum_{i=1}^{N-1} \frac{\eta_{i, 1}^{\prime \prime}+\cdots+\eta_{i, n}^{\prime \prime}}{n^{1 / p}} x_{i}\right)-E f\left(\sum_{i=1}^{N-1} \theta_{i} x_{i}\right)\right| \\
+\left|E f\left(\sum_{i=1}^{N-1} \theta_{i} x_{i}\right)-E f\left(\sum_{i=1}^{\infty} \theta_{i} x_{i}\right)\right|
\end{aligned}
$$

Let the index $\alpha$ satisfy $0<\alpha<p \wedge r \wedge 1$. The first term to the right of the inequality in (5.17) is less than or equal to

$$
E\left\|\sum_{i=N}^{\infty} \frac{\eta_{i, 1}^{\prime \prime}+\cdots+\eta_{i, n}^{\prime \prime}}{n^{1 / p}} x_{i}\right\|^{\alpha}
$$

and the third term is less than or equal to $E\left\|\sum_{i=N}^{\infty} \theta_{i} x_{i}\right\|^{\alpha}$. It follows from (5.16) and the remarks following (5.16) that each of these terms can be made arbitrarily small by taking $N$ sufficiently large. On the other hand, as $\boldsymbol{n}$ goes to infinity (for fixed $N$ ) the second term to the right of the inequality in (5.17) goes to zero. This is simply a finite dimensional result and each $\eta_{i}^{\prime \prime}$ is in the domain of attraction of $\boldsymbol{\theta}$.

Thus $Y^{\prime \prime}$ is in the domain of attraction of $Z$ and by hypothesis $Y^{\prime}$ is in the domain of attraction of $\delta_{0}$. It follows from Theorem 4.1 [34, p. 25] that $Y$ is 
in the domain of attraction of $Z$. This completes the proof of sufficiency.

In proving necessity we begin with the assumption that $Y$ is in the domain of normal attraction of $Z$. We write $Y=Y^{\prime}+Y^{\prime \prime}$ where $Y^{\prime}$ is any truncation of $Y$. Let $S_{n}^{\prime}$ and $S_{n}^{\prime \prime}$ be defined as above with respect to this truncation. It follows from the symmetry of the $\eta_{i}$ that $S_{n}^{\prime}+S_{n}^{\prime \prime}$ and $S_{n}^{\prime}-S_{n}^{\prime \prime}$ are equally distributed random vectors. Therefore, for any absolutely $r$-convex set $K$, $P\left[S_{n}^{\prime}+S_{n}^{\prime \prime} \notin K\right] \geqslant \frac{1}{2} P\left[S_{n}^{\prime} \notin 2^{1 / r-1} K\right]$. Since the measures corresponding to $S_{n}^{\prime}+S_{n}^{\prime \prime}$ are uniformly tight and since the closure of the absolute $r$-convexification of a compact set in $E$ is also compact, we see that the measures corresponding to $S_{n}^{\prime}$ are also uniformly tight. Now, since $\sum_{i=1}^{\infty} \eta_{i} x_{i}$ is in the domain of normal attraction of $\sum_{i=1}^{\infty} \theta_{i} x_{i}$, for any linear functional $x^{*} \in E^{*}$ we have that $\sum_{i=1}^{\infty} \eta_{i} x^{*} x_{i}$ is in the domain of normal attraction of $\sum_{i=1}^{\infty} \theta_{i} x^{*} x_{i}$. We will show that this implies that $\sum_{i=1}^{\infty} \eta_{i}^{\prime} x^{*} x_{i}$ is in the domain of normal attraction of $\delta_{0}$. Since this is true for all $x^{*}$ and since we know that laws of $S_{n}^{\prime}$ are uniformly tight we have that $S_{n}^{\prime}$ is in the domain of normal attraction of $\delta_{0}$.

Let $a_{i}=x^{*} x_{i}$. Thus $a_{i}$ is a real number. We now show that if $\sum_{i=1}^{\infty} \eta_{i} a_{i}$ is in the domain of normal attraction of $\sum_{i=1}^{\infty} \theta_{i} a_{i}$ then $\sum_{i=1}^{\infty} \eta_{i}^{\prime} a_{i}$ is in the domain of normal attraction of $\delta_{0}^{\prime}$, where $\delta_{0}^{\prime}$ is the unit mass at zero of the real line. Considering the relevant characteristic functions we see that, for all integers $N>1, \sum_{i=N}^{\infty} \eta_{i} a_{i}$ is in the domain of normal attraction of $\sum_{i=N}^{\infty} \theta_{i} a_{i}$. Therefore

$$
\lim _{a \rightarrow \infty} a^{p} P\left[\left|\sum_{i=N}^{\infty} \eta_{i} a_{i}\right|>a\right]=\frac{c_{p} \sum_{i=N}^{\infty}\left|a_{i}\right|^{p}}{a^{p}} .
$$

(This is a classical result. It can be readily derived using (5.5) and Theorem 1 [27] taking into account the fact that $\sum_{i=N}^{\infty} \theta_{i} a_{i}$ is equal in distribution to $\left(\sum_{i=N}^{\infty}\left|a_{i}\right|^{p}\right)^{1 / p} \theta$.)

Consider $\sum_{i=1}^{\infty} \eta_{i}^{\prime} a_{i}$. For each $N$, there exists an $M_{N}$ such that $\left|\sum_{i=1}^{N} \eta_{i}^{\prime} a_{i}\right|<$ $M_{N}$. Therefore

$$
\begin{aligned}
P\left[\left|\sum_{i=1}^{\infty} \eta_{i}^{\prime} a_{i}\right|>a\right] & \leq P\left[\left|\sum_{i=1}^{N-1} \eta_{i}^{\prime} a_{i}\right|>M_{N}\right]+P\left[\left|\sum_{i=N}^{\infty} \eta_{i}^{\prime} a_{i}\right|>-M_{N}\right] \\
& =P\left[\left|\sum_{i=N}^{\infty} \eta_{i}^{\prime} a_{i}\right|>a-M_{N}\right] .
\end{aligned}
$$

Note that $\Sigma \eta_{i}^{\prime} a_{i}+\sum \eta_{i}^{\prime \prime} a_{i}$ and $\Sigma \eta_{i}^{\prime} a_{i}-\sum \eta_{i}^{\prime \prime} a_{i}$ are equally distributed; therefore

$$
\begin{aligned}
P\left[\left|\sum_{i=N}^{\infty} \eta_{i}^{\prime} a_{i}\right|>a-M_{N}\right] & \leqslant 2 P\left[\left|\sum_{i=N}^{\infty} \eta_{i} x_{i}\right|>a-M_{N}\right] \\
& <\frac{2 c_{p} \sum_{i=N}^{\infty}\left|a_{i}\right|^{p}}{a^{p}\left(1-M_{N} / a\right)^{p}}
\end{aligned}
$$


Thus we have $\lim _{a \rightarrow \infty} a^{p} P\left[\left|\sum_{i=1}^{\infty} \eta_{i}^{\prime} a_{i}\right|>a\right]<K \sum_{i=N}^{\infty}\left|a_{i}\right|^{p}$ for some constant $K$ and since this holds for all $N$ we get $\lim _{a \rightarrow \infty} a^{p} P\left[\left|\sum_{i=1}^{\infty} \eta_{i}^{\prime} a_{i}\right|>a\right]=0$. Since the real line is of Rademacher type 2 we can use Theorem 3.1 to show that $\sum_{i=1}^{\infty} \eta_{i}^{\prime} a_{i}$ is in the domain of normal attraction of $\delta_{0}^{\prime}$. This completes the proof of Lemma 5.3.

The next result provides the "only if" part of Theorem 5.1(iii); however since it applies to all quasi-normed spaces we state it as a separate theorem.

THEOREM 5.4. Let $\theta,\left\{\theta_{i}\right\},\left\{x_{i}\right\}$ be as above and let $\left\{\eta_{i}\right\}$ be a sequence of independent symmetric real valued random variables. Assume that $\Sigma \theta_{i} x_{i}$ exists, $\sum \eta_{i} x_{i}$ exists and $\Sigma \eta_{i} x_{i}$ is in the domain of normal attraction of $\Sigma \theta_{i} x_{i}$. Then

$$
\lim _{a \rightarrow \infty} a^{p} P\left[\left\|\sum_{i=1}^{\infty} \eta_{i} x_{i}\right\|>a\right]=c_{p} \sum_{i=1}^{\infty}\left\|x_{i}\right\|^{p} .
$$

Note. This result holds for $\eta_{i}=\theta_{i}$; also $\Sigma \theta_{i} x_{i}$ exists implies $\Sigma\left\|x_{i}\right\|^{p}<\infty$.

Proof. By (1.9) we can obtain a linear functional $x^{*} \in E^{*}$ such that $x^{*}\left(\sum \eta_{i} x_{i}\right)=\eta_{i} x^{*} x_{i}$ and similarly $x^{*}\left(\sum \theta_{i} x_{i}\right)=\theta_{i} x^{*} x_{i}$. Thus $\eta_{i}$ is in the domain of normal attraction of $\theta$.

Since $Y=\sum_{i=1}^{\infty} \eta_{i} x_{i}$ is in the domain of normal attraction of $\sum_{i=1}^{\infty} \theta_{i} x_{i}$ it follows from Lemma 5.3 that all truncations $Y^{\prime}=\sum_{i=1}^{\infty} \eta_{i}^{\prime} x_{i}$ of $Y$ are in the domain of normal attraction of $\delta_{0}$. By (3.2) this implies that

$$
\lim _{a \rightarrow \infty} a^{p} P\left[\left\|Y^{\prime}\right\|>a\right]=0
$$

for all truncations $Y^{\prime}$ of $Y$. For a given $\varepsilon>0$ we choose the sequence $\left\{c_{i}\right\}$ established at the beginning of the proof of Lemma 5.3 and form the corresponding decomposition $Y=Y^{\prime}+Y^{\prime \prime}$, where $Y^{\prime \prime}=\sum_{i=1}^{\infty} \eta_{i}^{\prime \prime} x_{i}$ for $\eta_{i}^{\prime \prime}$ as defined in (5.8). Following Lemma 4.1 define $F(t)=P\left(\left\|\Sigma \eta_{i}^{\prime \prime} x_{i}\right\|_{r}>t\right), N=$ $\sup _{i}\left\|\eta_{i}^{\prime \prime} x_{i}\right\|_{r}$ and $G(t)=P(N>t)$. By Lemma 4.1 we have

$$
F(3 t) \leqslant G(t)+4 F^{2}(t)
$$

and by (5.10)

$$
G(t)<\sum_{i=1}^{\infty} P\left(\left\|\eta_{i}^{\prime \prime} x_{i}\right\|_{r}>t\right)<\frac{c_{p}(1+\varepsilon) \sum_{i=1}^{\infty}\left\|x_{i}\right\|^{p}}{t^{p / r}} .
$$

We will now show that there exists a $t_{0}$ such that

$$
F(t) \leqslant \frac{C \sum_{i=1}^{\infty}\left\|x_{i}\right\|^{p}}{t^{p / r}}
$$

for $t>t_{0}$ and for some fixed constant $C$. If $4 F^{2}(t)>\frac{1}{2} F(3 t)$ for all $t>t_{1}$ for some fixed $t_{1}>0$, then there exist numbers $\alpha>0, \beta>0, t_{2}>0$ such that for $t>t_{2}, F(t)<e^{-\alpha|t|^{\beta}}$ and this implies (5.23). Therefore we will assume that there exists a sequence $\left\{t_{k}\right\}, t_{k} \uparrow \infty$ such that $4 F^{2}\left(t_{k}\right)<\frac{1}{2} F\left(3 t_{k}\right)$. By (5.21) 
and (5.22) we have

$$
\begin{aligned}
F\left(3 t_{k}\right) & <2 G\left(t_{k}\right)<\frac{2 c_{p}(1+\varepsilon) \sum_{i=1}^{\infty}\left\|x_{i}\right\|^{p}}{t_{k}^{p / r}}, \\
F\left(9 t_{k}\right) & \leqslant G\left(3 t_{k}\right)+4 F^{2}\left(3 t_{k}\right) \\
& \leqslant \frac{c_{p}(1+\varepsilon) \sum_{i=1}^{\infty}\left\|x_{i}\right\|^{p}}{\left(3 t_{k}\right)^{p / r}}+\frac{16\left(c_{p}\right)^{2}(1+\varepsilon)^{2}\left(\sum_{i=1}^{\infty}\left\|x_{i}\right\|^{p}\right)^{2}}{t_{k}^{2 p / r}} .
\end{aligned}
$$

Therefore for some $t_{k_{0}}$ sufficiently large we have for $t_{k}>t_{k_{0}}$ that

$$
F\left(9 t_{k}\right)<\frac{2 c_{p}(1+\varepsilon) \sum_{i=1}^{\infty}\left\|x_{i}\right\|^{p}}{\left(3 t_{k}\right)^{p / r}} .
$$

Thus

$$
F\left(3 t_{k}\right)<\frac{2 c_{p}(1+\varepsilon) \sum_{i=1}^{\infty}\left\|x_{i}\right\|^{p}}{\left(3^{j-1} t_{k}\right)^{p / r}}
$$

Interpolating we get (5.23). We now sharpen the constant in (5.23). Using (5.22) and (5.23) in Lemma 4.1 with $s=(k-2) t$ we get

$$
F(k t)<\frac{c_{p}(1+\varepsilon) \sum_{i=1}^{\infty}\left\|x_{i}\right\|^{p}}{(k t)^{p / r}}\left[\left(\frac{k}{k-2}\right)^{p / r}+\frac{4 C^{2} \sum_{i=1}^{\infty}\left\|x_{i}\right\|^{p}}{c_{p}(1+\varepsilon)}\left(\frac{k}{t}\right)^{p / r}\right]
$$

for $t>t_{0}$ and all integers $k>3$. Let $s_{0}>t_{0}>10^{4}$ and $s_{0}^{5 / 4}+1<s<s_{0}^{7 / 4}$. Then there exists a $t>s_{0}$ and an integer $k, s_{0}^{1 / 4}<k<s_{0}^{3 / 4}$, such that $s=k t$. By (5.26) we have

$$
F(s)<\frac{c_{p}(1+\varepsilon) \sum_{i=1}^{\infty}\left\|x_{i}\right\|^{p}}{s^{p / r}}\left[1+\frac{4 p}{r s_{0}^{\prime \prime} 4}+\frac{4 C^{2} \sum_{i=1}^{\infty}\left\|x_{i}\right\|^{p}}{c_{p}(1+\varepsilon)}\left(\frac{1}{s_{0}}\right)^{p / 4 r}\right] .
$$

Note that as $s_{0}$ increases the bound on the right decreases. Thus we get

$$
\lim _{a \rightarrow \infty} a^{p} P\left[\left\|\sum_{i=1}^{\infty} \eta_{i}^{\prime \prime} x_{i}\right\|>a\right]<c_{p}(1+\varepsilon) \sum_{i=1}^{\infty}\left\|x_{i}\right\|^{p} .
$$

The value of $\varepsilon$ depends on the sequence $\left\{c_{i}\right\}$. Increasing all the $\left\{c_{i}\right\}$ allows us to decrease $\varepsilon$ without affecting (5.20).

The upper bound in (5.19) is obtained by the following argument:

$$
\begin{aligned}
P\left[\|Y\|_{r}>a^{r}\right] & =P\left[\left\|Y^{\prime}+Y^{\prime \prime}\right\|_{r}>a^{r}\right] \\
& <P\left[\left\|Y^{\prime \prime}\right\|_{r}>(a /(1+\delta))^{r}\right]+P\left[\left\|Y^{\prime}\right\|>a^{r}(r \delta)\right]
\end{aligned}
$$


for some small $\delta>0$. Thus

$$
\begin{aligned}
\lim _{a \rightarrow \infty} a^{p} P[\|Y\|>a]< & \lim _{a \rightarrow \infty} a^{p} P\left[\left\|Y^{\prime \prime}\right\|>a /(1+\delta)\right] \\
& +\lim _{a \rightarrow \infty} a^{p} P\left[\left\|Y^{\prime}\right\|>a(r \delta)^{1 / r}\right] \\
& <2(1+\varepsilon) \sum_{i=1}^{\infty}\left\|x_{i}\right\|^{p} .
\end{aligned}
$$

Since, with $\beta=(r \delta)^{1 / r}$,

$$
\lim _{a \rightarrow \infty} a^{p} P\left[\left\|Y^{\prime}\right\|>a \beta\right]=\beta^{-p} \lim _{a \rightarrow \infty} a^{p} P\left[\left\|Y^{\prime}\right\|>a\right]=0 .
$$

Since (5.28) holds for all $\varepsilon>0$ we obtain the upper bound in (5.19).

Obtaining the lower bound is easier. For a fixed value of $N$, to be specified below, let $a_{0}>\sup _{1<i<N} c_{i}(1+\delta)\left\|x_{i}\right\|^{-1}$. Then for $\delta>0$ sufficiently small and $a>a_{0}$

$$
\begin{aligned}
& P\left[\left\|\sum_{i=1}^{N} \eta_{i}^{\prime \prime} x_{i}\right\|_{r}>(a(1+\delta))^{r}\right] \\
& >\sum_{i=1}^{N}\left\{P\left[\left\|\eta_{i}^{\prime \prime} x_{i}\right\|_{r}>[a(1+\delta)]^{r}\right]\right. \\
& \left.\times P\left[\left\|\sum_{\substack{j=1 \\
j \neq i}} \eta_{j} x_{j}\right\|_{r}<r \delta a^{r}, \sup _{\substack{1<j<N \\
j \neq i}}\left\|\eta_{j}^{\prime \prime} x_{j}\right\|_{r}<[a(1+\delta)]^{r}\right]\right\} \\
& >\sum_{i=1}^{N} \frac{c_{p}(1-\varepsilon)\left\|x_{i}\right\|^{p}}{(1+\delta) a^{p}}\left\{1-P\left[\left\|\sum_{\substack{j=1 \\
j \neq i}}^{N} \eta_{j}^{\prime \prime} x_{j}\right\|_{r}>r \delta a^{r}\right]\right. \\
& \left.-\sum_{\substack{j=1 \\
j \neq i}}^{N} P\left[\left\|\eta_{j}^{\prime \prime} x_{j}\right\|_{r}>[a(1+\delta)]^{r}\right]\right\}
\end{aligned}
$$

By (5.27) we have

$$
P\left[\left\|\sum_{\substack{j=1 \\ j \neq i}}^{N} \eta_{j}^{\prime \prime} x_{j}\right\|_{r}>r \delta a^{r}\right]<\left(\frac{1}{r \delta}\right)^{1 / r} \frac{c_{p}(1+\varepsilon) \sum_{j=1}^{N}\left\|x_{i}\right\|^{p}}{a^{p}} .
$$

Also

$$
\sum_{\substack{j=1 \\ j \neq i}}^{N} P\left[\left\|\eta_{j}^{\prime \prime} x_{j}\right\|_{r}>[a(1+\delta)]^{r}\right]<\frac{c_{p}(1+\varepsilon) \sum_{j=1}^{N}\left\|x_{j}\right\|^{p}}{(1+\delta)^{p} a^{p}}
$$


It follows that

$$
\lim _{a \rightarrow \infty} a^{p} P\left[\left\|\sum_{i=1}^{N} \eta_{i}^{\prime \prime} x_{i}\right\|>a\right] \geqslant c_{p}(1-\varepsilon) \sum_{i=1}^{N}\left\|x_{i}\right\|^{p} .
$$

Next we note that for a sufficiently large

$$
\begin{gathered}
P\left[\left\|\sum_{i=1}^{\infty} \eta_{i}^{\prime \prime} x_{i}\right\|_{r}>a^{r}\right] \\
\geqslant P\left[\left\|\sum_{i=1}^{N} \eta_{i}^{\prime \prime} x_{i}\right\|_{r}>[(1+\delta) a]^{r}\right]-P\left[\left\|\sum_{i=N+1}^{\infty} \eta_{i}^{\prime \prime} x_{i}\right\|_{r}>r \delta a^{r}\right] \\
\geqslant \frac{c_{p}(1-2 \varepsilon) \sum_{i=1}^{N}\left\|x_{i}\right\|^{p}}{(1+\delta)^{p} a^{p}}-\frac{c_{p} \sum_{i=N+1}^{\infty}\left\|x_{i}\right\|^{p}}{(r \delta)^{1 / r} a^{p}}
\end{gathered}
$$

Now we choose $N$ so that the last term above is negligible with respect to the preceding term. Thus we get

$$
\lim _{a \rightarrow \infty} a^{P} P\left[\left\|\sum_{i=1}^{\infty} \eta_{i}^{\prime \prime} x_{i}\right\|>a\right] \geqslant c_{p}(1-4 \varepsilon) \sum_{i=1}^{N}\left\|x_{i}\right\|^{p}
$$

and since this holds for all $N$

$$
\lim _{a \rightarrow \infty} a^{p} P\left[\left\|\sum_{i=1}^{\infty} \eta_{i}^{\prime \prime} x_{i}\right\|>a\right]>c_{p}(1-4 \varepsilon) \sum_{i=1}^{\infty}\left\|x_{i}\right\|^{p} .
$$

We repeat the argument of (5.30) except this time we write $Y=Y^{\prime}+Y^{\prime \prime}$. Taking (5.20) into account we get

$$
\lim _{a \rightarrow \infty} a^{p} P\left[\left\|\sum_{i=1}^{\infty} \eta_{i}^{\prime \prime} x_{i}\right\|>a\right] \geqslant c_{p}(1-8 \varepsilon) \sum_{i=1}^{\infty}\left\|x_{i}\right\|^{p},
$$

and since this holds for all $\varepsilon>0$ we obtain the lower bound in (5.19).

Proof OF THEOREM 5.1. (i) $\Rightarrow$ (ii). This is Theorem 3.1.

(i) $\Rightarrow$ (iii). Since $E$ is of Rademacher type $p+\varepsilon, \varepsilon>0$, it is also of stable type $p$. Therefore we have that $\Sigma\left\|x_{i}\right\|^{p}<\infty$ implies $\Sigma \theta_{i} x_{i}$ exists. Also if we take the set $A$ in (5.2) to be a single integer we see that each $\eta_{i}$ is individually in the domain of attraction of $\theta$. In view of Lemma 5.3 and (ii) (since (i) also implies (ii)) it suffices to show that condition (5.2) implies that for any truncation $Y^{\prime}=\sum_{i=1}^{\infty} \eta_{i}^{\prime} x_{i}$ of $Y$ we have

$$
\lim _{a \rightarrow \infty} a^{p} P\left[\left\|\sum_{i=1}^{\infty} \eta_{i}^{\prime} x_{i}\right\|>a\right]=0
$$


Indeed, for each $N$ there exists an $M_{N}$ such that $\left\|\sum_{i=1}^{N-1} \eta_{i}^{\prime} x_{i}\right\|_{r}<M_{N}$. Thus

$$
\begin{aligned}
P\left[\left\|\sum_{i=1}^{\infty} \eta_{i}^{\prime} x_{i}\right\|_{r}\right. & \left.>a^{r}\right] \\
& <P\left[\left\|\sum_{i=1}^{N} \eta_{i}^{\prime} x_{i}\right\|_{r}>M_{N}\right]+P\left[\left\|\sum_{i=N}^{\infty} \eta_{i}^{\prime} x_{i}\right\|_{r}>a^{r}-M_{N}\right] \\
& =P\left[\left\|\sum_{i=N}^{\infty} \eta_{i}^{\prime} x_{i}\right\|_{r}>a^{r}-M_{N}\right] .
\end{aligned}
$$

Note that $\sum \eta_{i}^{\prime} x_{i}+\sum \eta_{i}^{\prime \prime} x_{i}$ and $\sum \eta_{i}^{\prime} x_{i}-\sum \eta_{i}^{\prime \prime} x_{i}$ are equally distributed. Therefore

$$
\begin{aligned}
P\left[\left\|\sum_{i=N}^{\infty} \eta_{i}^{\prime} x_{i}\right\|_{r}>a^{r}-M_{N}\right] & <2 P\left[\left\|\sum_{i=N}^{\infty} \eta_{i} x_{i}\right\|_{r}>a^{r}-M_{N}\right] \\
& <\frac{4 c_{p} \sum_{i=N}^{\infty}\left\|x_{i}\right\|^{p}}{a^{p}\left(1-M_{N} / a^{r}\right)^{p / r}} .
\end{aligned}
$$

Thus $\lim _{a \rightarrow \infty} a^{p} P\left[\left\|\sum_{i=1}^{\infty} \eta_{i}^{\prime} x_{i}\right\|>a\right]<K \sum_{i=N}^{\infty}\left\|x_{i}\right\|^{p}$ for some constant $K$ and since this holds for all $N$ we get (5.32). This completes the proof of (i) $\Rightarrow$ (iii).

(iii) $\Rightarrow$ (iv). Actually we show not (iv) implies not (iii). If $E$ is not of stable type $p$ there exists a sequence $\left\{x_{i}\right\}$ of elements in $E$ such that $\sum_{i=1}^{\infty}\left\|x_{i}\right\|^{p}<\infty$ but such that $\sum_{i=1}^{\infty} \theta_{i} x_{i}$ does not exist. For such a seqquence consider the series $\sum_{i=1}^{\infty} \eta_{i}^{\prime \prime} x_{i}$ where $\eta_{i}^{\prime \prime}$ are independent and

$$
\eta_{i}^{\prime \prime}= \begin{cases}0 & \text { if }\left|\theta_{i}\right|<c_{i} \\ \theta_{i} & \text { if }\left|\theta_{i}\right|>c_{i} .\end{cases}
$$

Here the sequence $c_{i}$ is chosen such that $\sum_{i=1}^{\infty} P\left[\left|\eta_{i}^{\prime \prime}\right| \neq 0\right]<\infty$. Thus, by the Borel-Cantelli lemma, $\sum_{i=1}^{\infty} \eta_{i}^{\prime \prime} x_{i}<\infty$ and defines an $E$-valued random vector. It follows from (5.27) and (5.31) that

$$
\lim _{a \rightarrow \infty} a^{p} P\left[\left\|\sum_{i \in A} \eta_{i}^{\prime \prime} x_{i}\right\|>a\right]=c_{p} \sum_{i \in A}\left\|x_{i}\right\|^{p}<\infty,
$$

since in both (5.27) and (5.31) the indexing of the terms and the values of the $\left\{x_{i}\right\}$ were completely arbitrary except for the assumption $\sum_{i=1}^{\infty} \eta_{i}^{\prime \prime} x_{i}$ exists. Neither of these statements depended upon the existence of $\sum_{i=1}^{\infty} \theta_{i} x_{i}$. Therefore $\sum_{i=1}^{\infty} \eta_{i}^{\prime \prime} x_{i}$ satisfies the left side of (5.2) but its law is not in the domain of attraction of any measure, since by checking the finite dimensional distributions we see that the limiting measure would have to have the distribution of $\sum_{i=1}^{\infty} \theta_{i} x_{i}$ and this series does not converge.

(ii) $\Rightarrow$ (iv). Again we actually show not (iv) implies not (ii). We will find a counterexample closely related to the one just used. Again we assume that $E$ 
is not of stable type $p$ and choose the same sequence $\left\{x_{i}\right\}$ for which $\Sigma \theta_{i} x_{i}$ does not converge a.s. By the extended Ito and Nisio theorem it does not converge in probability either. Therefore we can find an $\varepsilon>0$ and a sequence $N_{k} \uparrow \infty$ such that

$$
P\left[\left\|\sum_{i=N_{k}}^{N_{k+1}} \theta_{i} x_{i}\right\|>\varepsilon\right]>\varepsilon .
$$

Now consider $X^{k}=\sum_{i=N_{k}}^{N_{k+1}} \eta_{i}^{\prime \prime} x_{i}$ for $\eta_{i}^{\prime \prime}$ as defined in (5.34). Each $\eta_{i}^{\prime \prime}$ is individually in the domain of attraction of $\theta_{i}$ and since we are only considering a finite sum we can use characteristic functionals to see that

$$
\mathfrak{E}\left(\frac{X_{1}^{k}+\cdots+X_{m}^{k}}{m^{1 / P}}\right) \rightarrow \mathcal{E}\left(\sum_{i=N_{k}}^{N_{k+1}} \theta_{i} x_{i}\right)
$$

where $X_{1}^{k}, X_{2}^{k}, \ldots$ are independent copies of $X^{k}$. It follows that there exists an $m_{k}$ such that

$$
P\left[\left\|\sum_{i=N_{k}}^{N_{k+1}} \frac{\eta_{i, 1}^{\prime \prime}+\cdots+\eta_{i, m_{k}}^{\prime \prime}}{m_{k}^{1 / p}} x_{i}\right\|>\frac{\varepsilon}{2}\right]>\frac{\varepsilon}{2} .
$$

Now for $N_{k}<i<N_{k+1}$ let

$$
\rho_{i}= \begin{cases}\eta_{i}^{\prime \prime} & \text { if }\left|\eta_{i}^{\prime \prime}\right|<M_{k}, \\ 0 & \text { otherwise. }\end{cases}
$$

Let $\rho_{i, 1}, \rho_{i, 2}, \ldots$ be independent copies of $\rho_{i}$. Here we choose $M_{k}$ such that

$$
P\left[\rho_{i, j}=\eta_{i, j} ; i=N_{k}, \ldots, N_{k+1}, j=1, \ldots, m_{k}\right]>1-\varepsilon / 4 .
$$

Therefore, using (5.36) and (5.37) we get

$$
P\left[\left\|\sum_{i=N_{k}}^{N_{k+1}} \frac{\rho_{i, 1}+\cdots+\rho_{i, m_{k}}}{m_{k}^{1 / P}} x_{i}\right\|>\frac{\varepsilon}{2}\right]>\frac{\varepsilon}{4},
$$

and by our standard argument involving the sums of symmetric random variables we have

$$
P\left[\left\|\sum_{i=1}^{\infty} \frac{\rho_{i, 1}+\cdots+\rho_{i, m_{k}}}{m_{k}^{1 / p}} x_{i}\right\| \geqslant \frac{\varepsilon}{2}\right]>\frac{\varepsilon}{8} .
$$

Now $\sum_{i=1}^{\infty} \rho_{i} x_{i}$ converges a.s. for the same reason that $\sum_{i=1}^{\infty} \eta_{i}^{\prime \prime} x_{i}$ converges a.s. We see by (5.38) that $\sum_{i=1}^{\infty} \rho_{i} x_{i}$ does not satisfy the weak law of large numbers. Also each $\rho_{i}$ is a truncation of $\eta_{i}^{\prime \prime}$ and $\Sigma \eta_{i}^{\prime \prime} x_{i}$ satisfies (5.35). Therefore the same argument used to show that (5.2) implies (5.32) gives us $\lim _{a \rightarrow \infty} a^{p} P\left[\left\|\sum_{i=1}^{\infty} \rho_{i} x_{i}\right\|>a\right]=0$. Thus $\sum_{i=1}^{\infty} \rho_{i} x_{i}$ provides the counterexample. 
(ii) $\Rightarrow$ (iii). Since (ii) $\Rightarrow$ (iv) we have both (5.1) and the fact that $E$ is of stable type $p$. The proof of (i) $\Rightarrow$ (iii) uses precisely these two facts. This completes the proof of (i) $\Rightarrow$ (ii) $\Rightarrow$ (iii) $\Rightarrow$ (iv).

When $E$ is a Banach space the implication (iv) $\Rightarrow$ (i) is one of the major results of [22]. For $l_{r}, 0<r<1$, (iv) $\Rightarrow$ (i) is elementary.

6. Additional results and examples concerning the central limit theorem in quasi-normed spaces. Despite the critical role of spaces of stable type $p$ in Theorem 5.1, when the $\eta_{i}$ 's are also identically distributed we have a central limit theorem in all quasi-normed spaces.

THEOREM 6.1. Let $\theta$ be a real valued, symmetric random variable with characteristic function exp $-|t|^{p}, \eta$ be a real valued symmetric random variable which is in the domain of normal attraction of $\theta$, and $\left\{\theta_{i}\right\}$ and $\left\{\eta_{i}\right\}$ be independent copies of $\theta$ and $\eta$. If $\left\{x_{i}\right\} \subset E$ is such that $\sum_{i=1}^{\infty} \theta_{i} x_{i}$ exists, then $\sum_{i=1}^{\infty} \eta_{i} x_{i}$ exists and is in the domain of normal attraction of $\sum_{i=1}^{\infty} \theta_{i} x_{i}$.

Proof. Since the $\left\{\eta_{i}\right\}$ are identically distributed for every $\varepsilon>0$ one can find a $c>0$ such that

$$
c_{p}(1-\varepsilon) / t^{p} \leqslant P\left[\left|\eta_{i}\right|>t\right] \leqslant c_{p}(1+\varepsilon) / t^{p}
$$

for all $t \geqslant c$ (see (5.10)). Comparing $\eta_{i}$ to $2 \theta_{i}$ in (4.3) we see that Theorem 4.4 implies that $\Sigma \eta_{i} x_{i}$ converges a.s. Define $\eta_{i}=\eta_{i}^{\prime}+\eta_{i}^{\prime \prime}$ with respect to $c_{i}=c$ as in (5.8). Define $Y^{\prime}$ and $Y^{\prime \prime}$ as in the remarks following (5.8). In the proof of Lemma 5.3 we showed that $Y^{\prime \prime}$ is in the domain of normal attraction of $\sum_{i=1}^{\infty} \theta_{i} x_{i}$. Considering the remarks at the end of Lemma 5.3 we need only show that $Y^{\prime}=\sum_{i=1}^{\infty} \eta_{i}^{\prime \prime} x_{i}$ is in the domain of normal attraction of $\delta_{0}$. Note that $\eta_{i}^{\prime}$ are uniformly bounded.

Let $\left\{\rho_{i}\right\}$ be independent normal random variables with mean zero and variance 1. It follows from Theorem 4.4 that $\sum_{i=1}^{\infty} \rho_{i} x_{i}<\infty$. Therefore by Theorem 4.1 [19]

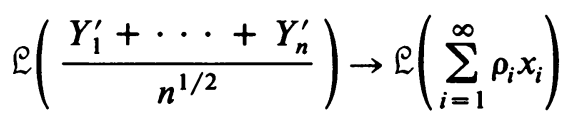

where $Y_{1}^{\prime}, Y_{2}^{\prime}, \ldots$, are independent copies of $Y^{\prime}$. (Actually Theorem 4.1 of [19] is proved only for continuous processes in Banach spaces; however the result holds in this case also. This is because (4.17) [19] enables us to use the argument of (5.16) of this paper and what follows to obtain (6.1).) Since $p<2$ we see that $Y^{\prime}$ is in the domain of normal attraction of $\delta_{0}$ and this completes the proof.

EXAMPLE 6.1. At the other extreme of Theorem 6.1 we now show that given any series $\sum_{i=1}^{\infty} \theta_{i} x_{i}$ as above in any quasi-normed space $E$ there exists a series $\sum_{i=1}^{\infty} \eta_{i} x_{i}$ such that each $\eta_{i}$ is individually in the domain of normal attraction 
of $\theta$ but $\sum_{i=1}^{\infty} \eta_{i} x_{i}$ is not in the domain of normal attraction of $\sum_{i=1}^{\infty} \theta_{i} x_{i}$ (both series exist).

By Lemma 5.3 it is enough to find a sequence of independent random variables $\left\{\xi_{i}\right\}$ such that $\left|\xi_{i}\right|<c_{i}, \sum_{i=1}^{\infty} \xi_{i} x_{i}$ exists but $\sum_{i=1}^{\infty} \xi_{i} x_{i}$ is not in the domain of normal attraction of $\delta_{0}$. Define

$$
\xi_{i}= \begin{cases}0 & \text { with probability } 1-p_{i}, \\ \pm \frac{i^{1 / p}}{\left\|x_{i}\right\|} & \text { each with probability } p_{i} / 2\end{cases}
$$

where $p_{i}=i^{-3 / 2}$. If $\sum_{i=1}^{\infty} \xi_{i} x_{i}$ is in the domain of normal attraction of $\delta_{0}$ then

$$
\lim _{m \rightarrow \infty} P\left[\left\|\sum_{i=m}^{\infty} \xi_{i}^{(m)} x_{i}\right\|>2^{1-1 / r}\right]=0
$$

where $\xi_{i}^{(m)}=\left(\xi_{i, 1}+\cdots+\xi_{i, m}\right) / m^{1 / p}$, the $\xi_{i, j}$ being independent copies of $\xi_{i}$. Let

$$
\gamma_{i, m}= \begin{cases}\xi_{i, j} & \text { if } \xi_{i, j} \neq 0 \text { and } \xi_{i, k}=0, k=1, \ldots, j-1, j+1, \ldots, m, \\ 0 & \text { otherwise. }\end{cases}
$$

Thus $\gamma_{i, m}= \pm i^{1 / p}\left\|x_{i}\right\|^{-1}$ each with probability $\frac{1}{2} m p_{i}\left(1-p_{i}\right)^{m-1}$. Define $\gamma_{i, m}^{\prime}$ $=\xi_{i}^{(m)} m^{1 / p}-\gamma_{i, m}$. Note that $\gamma_{i, m}^{\prime}+\gamma_{i, m}$ has the same distribution as $\gamma_{i, m}^{\prime}-$ $\gamma_{i, m}$. Therefore

or equivalently

$$
P\left[\left|\xi_{i}^{(m)} m^{1 / p}\right|>2^{1-1 / r}\right] \geqslant \frac{1}{2} P\left[\left|\gamma_{i, m}\right|>1\right]
$$

$$
P\left[\left\|\xi_{i}^{(m)}\right\|>2^{1-1 / r}\right]>\frac{1}{2} P\left[\left\|\left(\gamma_{i, m} / m^{1 / p}\right) x_{i}\right\|>1\right] .
$$

Therefore

$$
\begin{aligned}
P\left[\sup _{m<i}\left\|\xi_{i}^{(m)} x_{i}\right\|<2^{1-1 / r}\right] & =\prod_{i=m}^{\infty}\left[1-P\left(\left\|\xi_{i}^{(m)} x_{i}\right\|>2^{1-1 / r}\right)\right] \\
& <\prod_{i=m}^{\infty}\left[1-\frac{1}{2} P\left[\left\|\frac{\gamma_{i, m}}{m^{1 / p}} x_{i}\right\|>1\right]\right] .
\end{aligned}
$$

For $i>m,\left\|\left(\gamma_{i, m} / m^{1 / p}\right) x_{i}\right\|=i^{1 / p} / m^{1 / p}>1$ with probability $m p_{i}\left(1-p_{i}\right)^{m-1}$ $>m p_{i} / 2$ for $i, m>10$. Therefore

$$
P\left[\sup _{i}\left\|\xi_{i}^{(m)} x_{i}\right\|<2^{1-1 / r}\right]<\exp \left(-\frac{1}{4} \sum_{i=m}^{\infty} \frac{m}{i^{3 / 2}}\right)<\frac{1}{2}
$$

for $m$ sufficiently large. Now by symmetry and independence

$$
P\left[\left\|\sum_{i=m}^{\infty} \xi_{i}^{(m)} x_{i}\right\|>2^{1-1 / r}\right]>\frac{1}{2} P\left[\sup _{m<i}\left\|\xi_{i}^{(m)} x_{i}\right\|>2^{1-1 / r}\right]
$$


and using (6.3) we get

$$
P\left[\left\|\sum_{i=m}^{\infty} \xi_{i}^{(m)} x_{i}\right\|>2^{1-1 / r}\right] \geq \frac{1}{4}
$$

which contradicts (6.2).

We have been studying series of the form $Y=\sum_{i=1}^{\infty} \eta_{i} x_{i}$ where $x_{i} \in E, E$ a quasi-normed space and the $\left\{\eta_{i}\right\}$ are independent, symmetric, real valued random variables such that each $\eta_{i}$ is in the domain of attraction of $\theta$, a stable random variable with characteristic function $\exp -|t|^{p}, 0<p<2$. We have investigated the question of whether $\left(n^{-1 / p}\left(Y_{1}+\cdots+Y_{n}\right)\right)$ converges weakly. It is clear that if it does then the limit measure must be the law of $\sum_{i=1}^{\infty} \theta_{i} x_{i}$ where $\left\{\theta_{i}\right\}$ are independent copies of $\theta$. If $\sum_{i=1}^{\infty} \theta_{i} x_{i}$ does not exist (i.e., the partial sums do not converge a.s.) then $\mathcal{L}\left(n^{-1 / p}\left(Y_{1}+\cdots+Y_{n}\right)\right)$ can not converge weakly. Adopting the terminology used in the study of the conventional central limit theorem we say that $\sum_{i=1}^{\infty} \eta_{i} x_{i}$ is not prestable if $\sum_{i=1}^{\infty} \theta_{i} x_{i}$ does not exist. The counterexamples used in Theorem 5.1 to show not (iv) implies not (ii) and not (iv) implies not (iii) were both of this kind, i.e., the measures introduced were not prestable. In our final example we present prestable measures that satisfy hypothesis (5.2) of Theorem 5.1, but which do not satisfy the central limit theorem.

EXAMPLE 6.2. Consider the space $l^{q}$ with the usual $q$-homogeneous norm $\|z\|_{q}=\sum_{i=1}^{\infty}\left|z_{i}\right|^{q}$ for $z=\left(z_{1}, z_{2}, \ldots\right) \in l^{q}$ and quasi-norm $\|z\|=\|z\|_{q}^{1 / q}$. We show the following: for $0<q<p<2$, there exists $x_{i} \in l^{q}, i=$ $1,2, \ldots$, such that $\sum_{i=1}^{\infty} \eta_{i} x_{i}$ exists in $l^{q}, Z=\sum_{i=1}^{\infty} \theta_{i} x_{i}$ exists in $l^{q}$ and

$$
\lim _{a \rightarrow \infty} a^{p} P\left[\left\|\sum_{i \in A} \eta_{i} x_{i}\right\|>a\right]=c_{p} \sum_{i \in A}\left\|x_{i}\right\|^{p}
$$

(here $\left\{\eta_{i}\right\}$ and $\left\{\theta_{i}\right\}$ are as above), but $\left(n^{-1 / p}\left(Y_{1}+\cdots+Y_{n}\right)\right)$ does not converge weakly. These examples are consistent with our previous results because $l^{q}$ is not of stable type $p$ for $p>q$.

Let $x_{i}=\left(0, \ldots, 0, y_{i}, 0, \ldots\right)$ where $y_{i}=i^{-2 / q}$. We have $\sum_{i=1}^{\infty}\left\|x_{i}\right\|^{p}=$ $\sum_{i=1}^{\infty} i^{-2 p / q}<\infty$ since $p>q$. Also since $p>q, E|\theta|^{q}<\infty$ and we have $E\left[\left\|\sum_{i=1}^{\infty} \theta_{i} x_{i}\right\|_{q}\right]=E\left[\sum_{i=1}^{\infty}\left|\theta_{i}\right|^{q_{i}-2}\right]<\infty$. Therefore $\sum_{i=1}^{\infty} \theta_{i} x_{i}$ defines a stable measure on $l^{q}$.

Let $\left\{\rho_{i}\right\}$ be a sequence of independent random variables such that

$$
\rho_{i}= \begin{cases} \pm y_{i}^{-1} & \text { each with prob. }\left(2 i^{1+e}\right)^{-1}, \\ 0 & \text { otherwise, }\end{cases}
$$

where $\varepsilon=(1-q / p) / 4$. It is clear that $X=\sum_{i=1}^{\infty} \rho_{i} x_{i}$ exists since $\sum_{i=1}^{\infty} P\left(\rho_{i} \neq\right.$ $0)<\infty$. Note that $\left|\rho_{i} y_{i}\right|=1$ or 0 so $\left|\rho_{i} y_{i}\right|^{q}=\left|\rho_{i} y_{i}\right|$. Using this fact and the Chebyshev inequality we have 


$$
\begin{aligned}
P\left[\left\|\sum_{i=1}^{\infty} \rho_{i} x_{i}\right\|>a\right] & \leqslant \exp \left(-a^{q}\right) E\left[\exp \left(\sum_{i=1}^{\infty}\left|\rho_{i} x_{i}\right|\right)\right] \\
& \leqslant \exp \left(-a^{q}\right) C(\varepsilon)
\end{aligned}
$$

where $C(\varepsilon)$ is a constant depending upon $\varepsilon$. It follows that

$$
\lim _{a \rightarrow \infty} a^{p} P\left[\left\|\sum_{i \in A} \rho_{i} x_{i}\right\|>a\right]=0 .
$$

Let $\left\{\theta_{i}\right\}$ be independent copies of $\theta$ such that $\left\{\theta_{i}\right\}$ and $\left\{\rho_{i}\right\}$ are also mutually independent. We define $\eta_{i}=\rho_{i}+\theta_{i}$. Clearly $Y=\sum_{i=1}^{\infty} \eta_{i} x_{i}$ exists and each $\eta_{i}$ is in the domain of normal attraction of $\theta$. It follows from (6.6) and Theorem 5.4 that (6.4) is satisfied. Also since $\left\{\rho_{i}\right\}$ and $\left\{\theta_{i}\right\}$ are independent, by considering characteristie functionals we see that $\sum_{i=1}^{\infty} \eta_{i} x_{i}$ is in the domain of normal attraction of $\sum_{i=1}^{\infty} \theta_{i} x_{i}$ if and only if $X=\sum_{i=1}^{\infty} \rho_{i} x_{i}$ is in the domain of normal attraction of $\delta_{0}$. We now show that

$$
\mathcal{E}\left(\left(X_{1}+\cdots+X_{n}\right) / n^{1 / p}\right) \nrightarrow \delta_{0}, \quad n \rightarrow \infty,
$$

where $X_{1}, X_{2}, \ldots$ are independent copies of $X$. To do this we use Theorem $3.1[16]$.

Let $\left\{\xi_{i}\right\}$ be identically distributed, independent random variables with characteristic function exp $-|t|^{q}$ such that $\left\{\xi_{i}\right\}$ and $\left\{\rho_{i}\right\}$ are mutually independent. (Note this is the same $q$ as in $l^{q}$.) Let $\left(\Omega_{1}, \mathscr{F}_{1}, P_{1}\right)$ be the infinite product space generated by $\left\{\xi_{i}\right\}$ and let $\xi=\left(\xi_{1}, \xi_{2}, \ldots\right)$. It follows from Theorem 3.1 [16] that a necessary condition for the law of $S_{n}$ to converge weakly to $\delta_{0}$ is that

$$
E\left[e^{i\left\langle\xi, S_{n}\right\rangle}\right] \rightarrow 1,
$$

a.s. with respect to $P_{1}$ measure on $\Omega_{1}$, where, in our case $S_{n}=n^{-1 / p}\left(X_{1}\right.$ $\left.+\cdots+X_{n}\right)$. (Expectation is taken with respect to $S_{n}$.) We will conclude our example by contradicting (6.8).

By an easy computation we see that

$$
E\left[e^{i\left\langle\xi, S_{n}\right\rangle}\right]=\prod_{i=1}^{\infty}\left[1-i^{-(1+\varepsilon)}\left[1-\cos \xi_{i} / n^{1 / p}\right]\right]^{n} .
$$

Thus to contradict (6.8) it is enough to show that

$$
U_{n} \equiv \sum_{i=1}^{\infty} n i^{-(1+\varepsilon)}\left[1-\cos \frac{\xi_{i}}{n^{1 / p}}\right] \nrightarrow 0
$$

$P_{1}$-a.s. as $n \rightarrow \infty$. We have

$$
U_{n} \geqslant \frac{n}{4} \sum_{i=1}^{\infty} i^{-(1+\varepsilon)} \frac{\xi_{i}^{2}}{n^{2 / p}} I_{\left[\xi_{i}<n^{1 / p}\right]}
$$


For $n>n_{0}$ for some integer $n_{0}$

$$
\frac{\delta^{\prime}(q)}{n^{q / p}} \leqslant P\left[\frac{1}{2}<\frac{\left|\xi_{i}\right|}{n^{1 / p}}<1\right]<\frac{\delta^{\prime \prime}(q)}{n^{q / p}}
$$

where $\delta^{\prime}(q)=c_{q}\left(1-\varepsilon^{\prime}\right)\left(2^{q}-1\right), \delta^{\prime \prime}(q)=c_{q}\left(1+\varepsilon^{\prime}\right)\left(2^{q}-1\right)$ for some $\varepsilon^{\prime}>0$ which can be made arbitrarily small depending on $n_{0}$. Define $\left\{\varphi_{i}\right\}$ and $Y_{n}$ as follows:

$$
\varphi_{i}= \begin{cases}\left(\left|\xi_{i}\right| / n^{1 / p}\right)^{2} & \text { if } \frac{1}{2}<\left|\xi_{i}\right| / n^{1 / p}<1 \\ 0 & \text { otherwise }\end{cases}
$$

and

$$
Y_{n}=\frac{n}{4} \sum_{i=n}^{\infty} i^{-(1+\varepsilon)} \varphi_{i}
$$

Note that $U_{n}$ and $Y_{n}$ are both defined on $\left(\Omega_{1}, \mathscr{F}_{1}, P_{1}\right)$ and that $U_{n}>Y_{n}$ pointwise on $\Omega_{1}$. Furthermore, $\frac{1}{4}<\varphi_{i}<1$ on a set of measure equal to $\delta(q) / n^{q / p}$ where $\delta^{\prime}(q)<\delta(q)<\delta^{\prime \prime}(q)$, otherwise $\varphi_{i}=0$. We use the wellknown inequality $[12$, p. 6]

$$
P\left(Y_{n}>\lambda E Y_{n}\right) \geqslant(1-\lambda)^{2} E^{2} Y_{n} / E Y_{n}^{2}
$$

where $0<\lambda<1$. Since $\delta^{\prime}(q) / 4 n^{q / p}<E \varphi_{i}<\delta^{\prime \prime}(q) / n^{q / p}$ and $E \varphi_{i}^{2}<$ $\delta^{\prime \prime}(q) / n^{q / p}$ we get

$$
\frac{\delta^{\prime}(q, p)}{64} n^{(3 / 4)(1-q / p)}<E Y_{n}<\frac{\delta^{\prime \prime}(q, p)}{16} n^{(3 / 4)(1-q / p)}
$$

where $0<\delta^{\prime}(q, p)<\delta^{\prime \prime}(q, p)<\infty$. Also

$$
E Y_{n}^{2}<\left(E Y_{n}\right)^{2}+E\left[\frac{n^{2}}{16} \sum_{i=n}^{\infty} i^{-2(1+\varepsilon)} \varphi_{i}^{2}\right]
$$

and

$$
E\left[\frac{n^{2}}{16} \sum_{i=n}^{\infty} i^{-2(1+\varepsilon)} \varphi_{i}^{2}\right]<\delta(p, q) n^{(1 / 2)(1-q / p)}
$$

where $\delta(q, p)<\infty$ is a constant depending on both $q$ and $p$. Since $p>q$, we can substitute (6.12) and (6.11) into (6.10) to see that, as $n \rightarrow \infty, Y_{n}$ is unbounded on a set of positive measure. Since $U_{n}>Y_{n}$ pointwise we have obtained (6.9). This completes the demonstration.

Consider $X=\sum_{i=1}^{\infty} \rho_{i} x_{i}$. This satisfies condition (ii) of Theorem 5.1, yet $X$ does not satisfy the weak law of large numbers, as we have just shown. 


\section{REFERENCES}

1. A. Beck, $A$ convexity condition in Banach spaces and the strong law of large numbers, Proc. Amer. Math. Soc. 13 (1962), 329-334.

2. J. Bretagnolle, D. Dacunha-Castelle and J. L. Krivine, Lois stables et espaces $L^{p}$, Ann. Inst. H. Poincaré Sect. 2B (1966), 231-259.

3. I. Csiszar and B. S. Rajput, A convergence of types theorem for probability measures on topological vector spaces with application to stable laws, Z. Wahrscheinlichkeitstheorie und Verw. Gebiete 36 (1976), 1-7.

4. J. L. Doob, Stochastic processes, Wiley, New York, 1950.

5. R. M. Dudley and M. Kanter, Zero-one laws for stable measures, Proc. Amer. Math. Soc. 45 (1974), 245-252.

6. B. V. Gnedenko, On the theory of domains of attraction of stable laws, Učen. Zap. Moskov. Gos. Univ. Mat. 30 (1939), 61-81.

7. J. Hoffmann-Jørgensen, Sums of independent Banach space valued random variables, Aarhus Universitet Preprint Series 15 (1972/73), 1-89.

8. Sums of independent Banach space valued random variables, Studia Math. 52 (1974), 159-186.

9. J. Hoffmann-Jørgensen and G. Pisier, The law of large numbers and the central limit theorem in Banach spaces, Ann. Probability 4 (1976), 587-599.

10. N. C. Jain and M. B. Marcus, Integrability of infinite sums of independent vector-valued random variables, Trans. Amer. Math. Soc. 212 (1975), 1-36.

11. M. B. Marcus, Uniform convergence of random Fourier series, Ark. Mat. 13 (1975), 107-122.

12. J. P. Kahane, Some random series of functions, Heath, Lexington, Mass., 1968.

13. M. Klosowska, The domain of attraction of a non-gaussian stable distribution in a Hilbert space, Colloq. Math. 32 (1972), 127-136.

14. A. A. Kolmogorov, Bemerkungen zu meiner Arbeit "Über die Summen zufälliger Grössen", Math. Ann. 102 (1929), $484-488$.

15. J. L. Krivine, Sous-espace de dimension finie des espaces de Banach réticulés, Ann. Math. 104 (1976), 1-29.

16. J. Kuelbs and V. Mandrekar, Harmonic analysis on certain vector spaces, Trans. Amer. Math. Soc. 149 (1970), 213-231.

17. __ Domains of attraction of stable measures on a Hilbert space, Studia Math. 59 (1974), 149-162.

18. S. Kwapien, Sums of independent Banach space valued random variables (after J. HoffmannJorgensen), Séminaire Maurey-Schwartz 1972/73, Exposé VI.

19. M. B. Marcus, Some new results on central limit theorems for $C(S)$-valued random variables, Probability in Banach spaces, Lecture Notes in Math., vol. 526, Springer-Verlag, Berlin, 1976, pp. 167-186.

20. M. B. Marcus and W. A. Woyczynski, Domaines d'attraction normale dans l'espaces de type stable, C. R. Acad. Sci. Paris Sér. A-B 285 (1977), 915-917.

21. B. Maurey, Espaces de cotype p, $0<p<2$, Séminaire Maurey-Schwartz 1972/73, Exposé VII.

22. B. Maurey and G. Pisier, Séries de variables aléatoires vectorielles independantes et propriétés géométriques des espaces de Banach, Studia Math. 58 (1976), 45-90.

23. D. Mouchtari, Sur l'existence d'une topologie du type de Sazonov sur une espace de Banach, Séminaire Maurey-Schwartz 1975/76, Exposé XVII.

24. V. V. Petrov, Sums of independent random variables, "Nauka", Moscow, 1972. (Russian)

25. G. Pisier, Une propriété du type p-stable, Séminaire Maurey-Schwartz 1973/74, Exposé VIII.

26. __ Sur les espaces qui ne contiennent pas de $l_{n}^{1}$ uniformement, C. R. Acad. Sci. Paris Sér. A-B 277 (1973), 991-994.

27. E. J. G. Pitman, Some theorems on characteristic functions of probability distributions, Proc. Fourth Berkeley Symposium, vol. II, Univ. of California Press, Berkeley, 1961, pp. 393-402. 
28. E. L. Rvaceva, On domains of attraction of multidimensional distributions, Theory Probability and Math. Statist. 2 (1962), 183-205.

29. A. Tortrat, Sur les lois $\mathbf{e}(\lambda)$ dans les espaces vectorielles, Applications aux lois stables, $\mathbf{Z}$. Wahrscheinlichkeitstheorie und Verw. Gebiete 27 (1976), 175-182.

30. K. Urbanik and W. A. Woyczynski, Random integrals and Orlicz spaces, Bull. Acad. Polon. Sci. Sér. Sci. Math. Astronom. Phys. 15 (1967), 161-169.

31. W. A. Woyczynski, Geometry and martingales in Banach spaces. I, Lecture Notes in Math., vol. 472, Springer-Verlag, Berlin, 1976, pp. 229-275; II, Probability on Banach spaces, Advances in Probability, vol. 4, Dekker, New York, 1978, pp. 267-517.

32. Weak convergence to a Gaussian measure of martingales in Banach spaces, Sympos. Math. 21 (1977), 319-331.

33. K. Yosida, Functional analysis, Springer-Verlag, New York, 1965.

34. P. Billingsley, Convergence of probability measures, Wiley, New York, 1968.

35. S. Rolewicz, Metric linear spaces, PWN, Warsaw, 1972.

Department of Mathematics, NorthWestern Universtty, Evanston, Iluinois 60201 (Current address of M. B. Marcus)

Current address (W. A. Woyczynski): Department of Mathematics, Cleveland State University, Cleveland, Ohio 44115 